NATIONAL AERONAUTICS AND SPACE ADMINISTRATION

Technical Report 32-1576

\title{
Dynamic Analysis of a System of Hinge-Connected Rigid Bodies With Nonrigid Appendages
}

\author{
Peter W. Likins
}

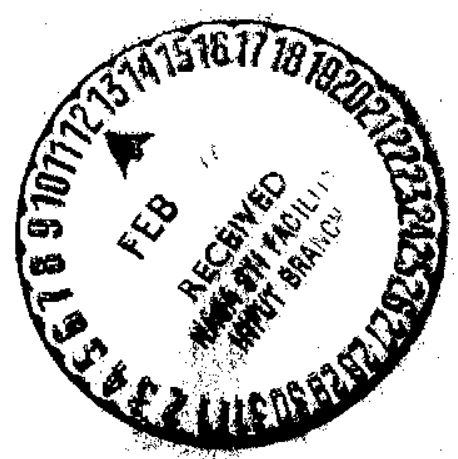

(MASA-CR-136627) DYNAMIC ANALYSIS OF A SYSTEM OF HINGE-CONNECTED RIGID BODIES

WTH NONRIGID ARPENDAGES (Jet Propulsion

Lab.) 29 p HC $\$ 3.50$ CSCL $20 \mathrm{~K}$

N $74-16588$

Unclas

G.3/32 27977

JET PROPULSION LABORATORY

CALIFORNIA INSTITUTE OF TECHNOLOGY

PASADENA, CALIFORNIA

February 1,1974 
NATIONAL AERONAUTICS AND SPACE ADMINISTRATION

Technical Report 32-1576

Dynamic Analysis of a System of Hinge-Connected
Rigid Bodies With Nonrigid Appendages

Peter W. Likins

JET PROPULSION LABORATORY

CALIFORNIA INSTITUTE OF TECHNOLOGY

PASADENA, CALIFORNIA

February 1, 1974 
Prepared Under Contract No. NAS $7 \cdot 100$

National Aeronautics and Space Administration 


\section{Preface}

The work described in this report was performed by the Guidance and Control Division of the Jet Propulsion Laboratory. 


\section{Contents}

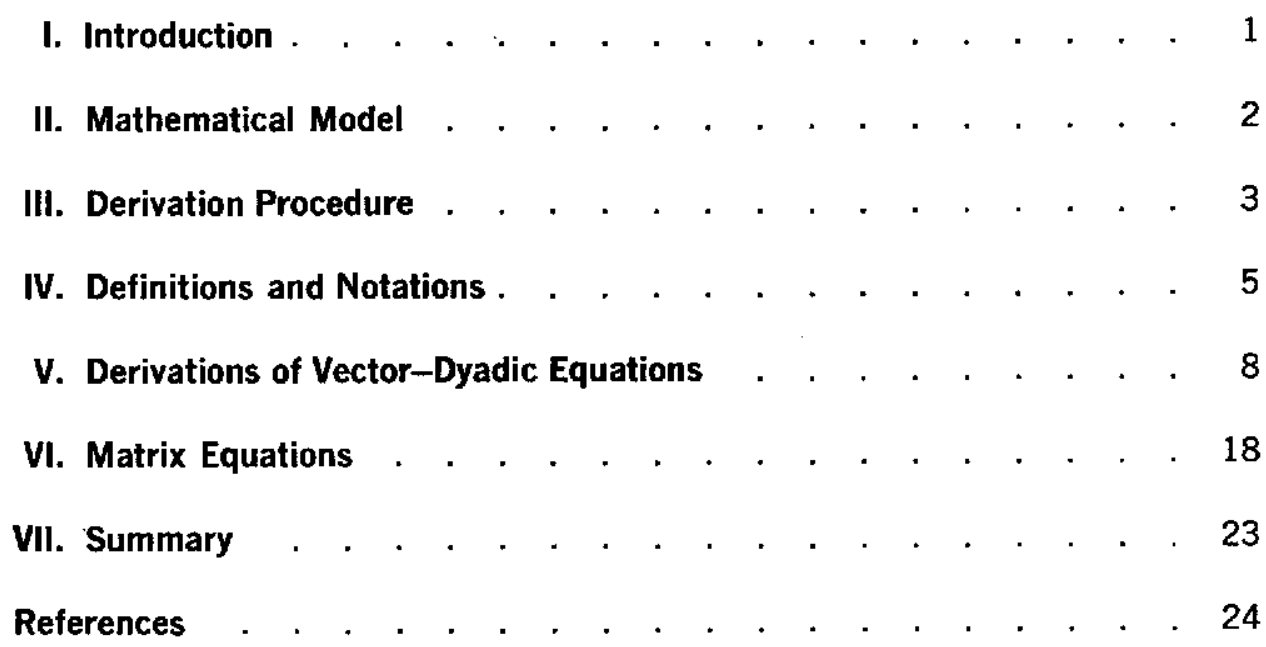

\section{Figures}

1. A spacecraft and its mathematical model . . . . . . . . . 4

2. Definitions for the $k$ th substructure, with $j<k$. . . . . . . . 5

3. System geometry . . . . . . . . . . . . . . . . 12

PRECEDIVG PAGE BLANE NOT FILMED 


\begin{abstract}
Equations of motion are derived for use in simulating a spacecraft or other complex electromechanical system amenable to idealization as a set of hinge-connected rigid bodies of tree topology, with rigid axisymmetric rotors and nonrigid appendages attached to each rigid body in the set. In conjunction with a previously published report on finite-element appendage vibration equations, this report provides a complete minimum-dimension formulation suitable for generic programming for digital computer numerical integration.
\end{abstract}




\section{Dynamic Analysis of a System of Hinge-Connected Rigid Bodies With Nonrigid Appendages}

\section{Introduction}

In a previously published report (Ref. 1), there appear equations of motion which charactcrize the small, time-varying deformations of an elastic appendage attached to a rigid body experiencing arbitrary motions in inertial space. The flexible appendage is modeled as a set of deformable elastic elements possessing distributed mass and interconnected at $n$ nodes, with a rigid nodal body appearing also at each node. This finite-element model has $6 n$ degrees of freedom in deformation, corresponding to the degrees of freedom invested in the nodal bodies; deformations of the internodal elastic elements are established by assigned interpolation functions. The purpose of Ref. 1 is to establish the structure of the $6 n$ deformation equations, in order to permit consideration of coordinate transformations which might introduce the possibility of coordinate truncation and the consequent representation of elastic appendage deformations in terms of distributed or modal coordinates numbering much less than $6 n$. With this objective accomplished, Ref. 1 terminates, leaving a set of equations of motion which are incomplete in the sense that they are insufficient to determine the kinematic variables characterizing the motion in inertial space of the rigid base to which the flexible appendage is attached.

It is the purpose of the present report not only to complete the dynamic analysis begun in the earlier work, but to do so in a way that encompasses a wide class of vehicles, namely, those amenable to idealization as a set of $n+1$ rigid bodies interconnected by $n$ line hinges (implying tree topology), with the possibility of rigid axisymmetric rotors and arbitrary nonrigid appendages attached to each rigid body in the set. 
The results of Ref. 1 provide the vibratory deformation equations for each elastic appendage in the system, and the transformation to distributed coordinates which is appropriate in each case. The new scalar equations to be derived in this paper number at least $n+6$, being descriptive of the inertial translations and rotations of one reference body and the relative rotations about the $n$ line hinges, with an additional equation being added for each axisymmetric rotor in the system. In this respect there is a strong parallel between the results of the present report and those obtained by extending the Hooker-Margulies equations (Ref. 2) as suggested by Hooker (Ref. 3) in order to eliminate unwanted kinematic constraint ${ }^{1}$ torques; the significant difference is that Hooker and Margulies considered only point-connected rigid bodies in a topological tree, whereas in the present report the basic elements in the tree are substructures which are rigid in part but include rotors and arbitrary nonrigid appendages.

A somewhat restricted version of these results is presented in Section VI of this report in a matrix form which has an affinity with the multiple-rigid-body formalism developed by Roberson and Wittenburg (Ref. 4) in chronological parallel with the derivation of the Hooker-Margulies equations (Ref. 2), although the form of the present equations lacks the aesthetic qualities of those in Ref. 4.

A digital computer program for the numerical integration of the general equations reported here is under development at the Jet Propulsion Laboratory by G. E. Fleischer. The result is to be a generic program, suitable for the dynamic simulation of a wide class of spacecraft. Many features of the following derivation have been adopted so as to minimize the labors of the users of this program.

\section{Mathematical Model}

Any problem of dynamic analysis must begin with the adoption of a mathematical model representing the physical system of interest. In what follows, it is assumed that the model consists of $n+1$ rigid bodies (labeled $b_{0}, \cdots, b_{n}$ ) interconnected by $n$ line hinges (implying no closed loops and hence tree topology), with each body containing an arbitrary number (perhaps zero) of rigid rotors, each with an axis of symmetry fixed in the housing body, and moreover with the possibility of attaching to each of the $n+1$ bodies a nonrigid appendage, with appendage $a_{k}$ attached to body $\mathfrak{b}_{k}$. The appendage itself can be modeled in a variety of ways without exceeding the scope of the final vector-dyadic equations in this paper; one might adopt a continuum model, a distributed-mass finite-element model, or a model admitting mass only in the form of nodal bodies or nodal particles.

Specific choices of appendage model are made only in Section VI of this report, where an explicit set of matrix equations is presented in order to illustrate the transition from generic vector-dyadic equations to the morc explicit matrix or scalar equations required for digital computer numerical integration. Although the equations derived here do not in their vector-dyadic form imply restricted appendage deformations, when specific appendage models are adopted it is assumed that their deformations relative to some reference state are "small" in the sense that terms above the first degree in deformation variables can be ignored.

\footnotetext{
IKinematic constraint forces and torques are respectively those interaction forces and torques which maintain kinematic constraints, such as "no relative translation of two points" or "no deviation from a prescribed relative rotation."
} 
If the actual connection between two massive portions of the physical system admits two (or three) degrees of freedom in rotation, then the analyst simply introduces one (or two) massless and dimensionless imaginary bodies into his model (as though they were massless gimbals). Since the number of equations to be derived here matches the number of degrees of freedom of the system, no price is paid in problem dimension by the introduction of imaginary bodies, and considerable simplification results in the user input format for the computer program.

Each combination of a rigid body and its internal rotors and attached flexible appendage comprises a basic building block referred to here as a substructure; thus, there are $n+\mathrm{I}$ substructures in the total system, so labeled that $a_{k}$ encompasses $\mathfrak{b}_{k}, \boldsymbol{a}_{k}$, and any rotors in $\mathfrak{b}_{k}$.

Figure 1 illustrates a typical spacecraft and its mathematical model; flexible appendages are cross-hatched. Note that the three physically distinct flexible structures attached to the central body are all combined as the single flexible appendage of $b_{0}$ in the mathematical model, and the two-degree-of-freedom gimbal joint of the midcourse motor has been accommodated with the introduction of an imaginary massless rigid body $\mathfrak{b}_{1}$. Because the fifth substructure encompasses the flexible antenna as well as the rigid body $b_{5}$, and because the mass properties to appear in the equations are those of the substructure (and not those of the rigid body), the mass properties of $b_{5}$ are irrelevant to the analysis. The masses of $b_{0}$ and the rotors within $\mathfrak{b}_{0}$ are also irrelevant, being absorbed in the mass of substructure $\boldsymbol{a}_{0}$.

Normally we must expect that the relative rotations between contiguous pairs of substructures will be governed by components of torque generated by clectric motors subject to control laws. It is this expectation that underlies the transition from the physical system to the mathematical model in Fig. 1. This is not a uniquely satisfactory model, however, and if attention were to focus on vehicle attitude control rather than on antenna pointing control, it might be desirable to replace the control systems governing the rotations between $b_{4}$ and $b_{5}$ and between $b_{4}$ and 60 by analytical expressions typical of elastic or viscoelastic connections. If this choice is made, it might be best to embrace the fourth and fifth substructures within the definition of the flexible appendage of $\boldsymbol{b}_{0}$. This decision would result in a "better" set of modal coordinates (permitting simulation with fewer variables), as one can appreciate after careful study of Ref. 1 . The importance of devising the most appropriate mathematical model for the task at hand deserves emphasis, since with the advent of automated derivation and integration of equations of motion this becomes the most important step in dynamic analysis.

\section{Derivation Procedure}

The derivation is described below.

Step 1. Isolate each substructure and apply

$$
\mathbf{F}_{T}^{j}=m_{j} \mathbf{A}^{j} \quad \text { and } \quad \mathbf{T}_{T}^{j}=\dot{\mathbf{H}}^{j} \quad i=0,1, \cdots, n
$$

where, for the $j$ th substructure, $\mathbf{F}_{T}^{j}$ is the total resultant of all external forces, $m_{j}$ is the substructure mass, $\mathbf{A}^{j}$ is the inertial acceleration of the substructure mass center $c_{j}, \mathbf{T}_{T}^{j}$ is the total moment resultant of all external forces referred to $c_{j}$, and $\dot{\mathbf{H}}^{j}$ is the incrtial time derivative of the substructure angular momentum referred to $c_{j}$. 


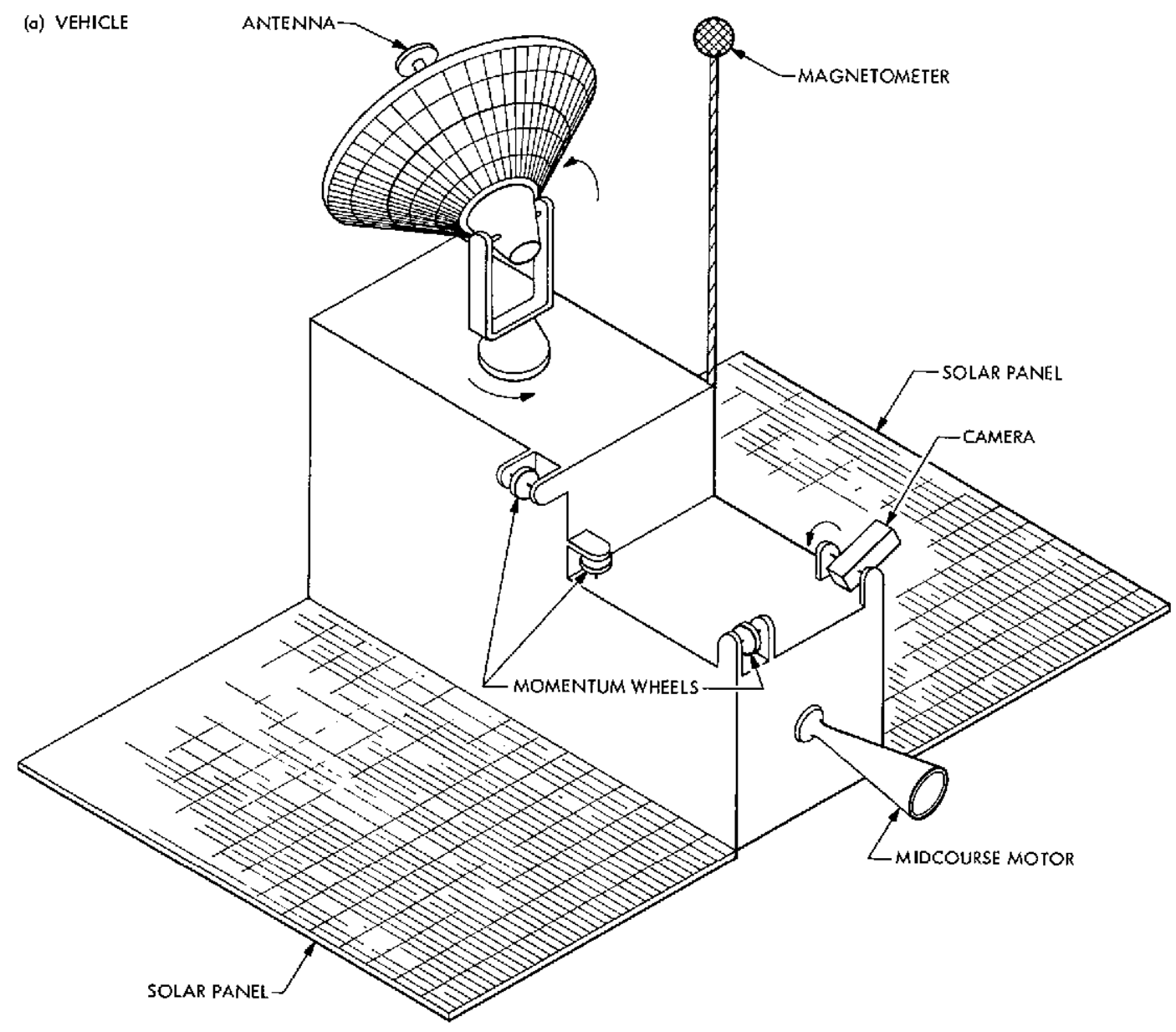

(b) IDEALIZATION

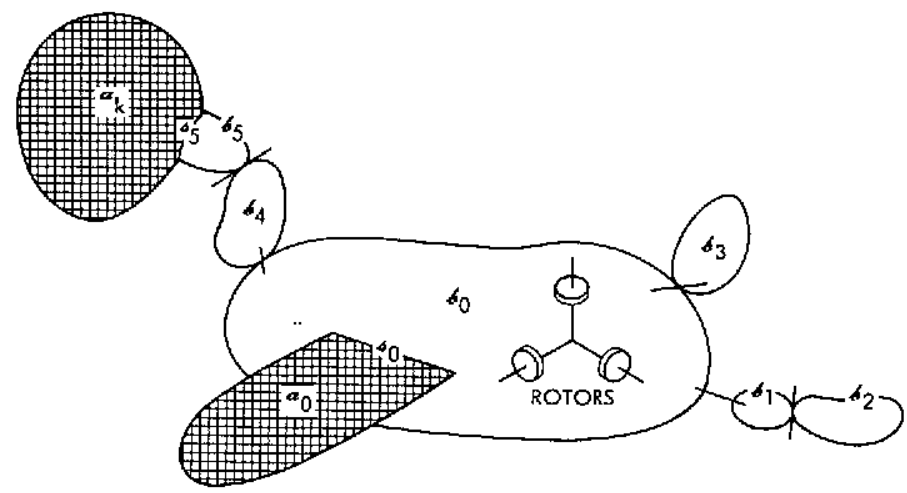

Fig. 1. A spacecraft and its mathematical model 
Step 2. Combine the $6 n+6$ scalar equations obtained in Step I so as to obtain $n+6$ scalar equations which do not involve redundant variables or those substructure interaction forces or torques which serve to maintain kinematic constraints.

Step 3. Apply $\mathbf{T}=\dot{\mathbf{H}}$ to each rotor of the system (where symbol definitions follow naturally from Eq. 1), and dot-multiply each equation by a unit vector parallel to the symmetry axis of the corresponding rotor; the result is a set of scalar equations matching in number the rigid, axisymmetric rotors in the system.

Step 4. Record the appendage deformation equations from Ref. 1 (or an alternative source, depending on the appendage model), and, if a finite-element model is used, substitute the deformation coordinate transformations from Ref. 1 wherever the deformation variables appear in the preceding equations. Maximum generality is retained in this report by postponing such substitutions until the equations are in otherwise final form, suitable for any appendage model.

Step 5. Specify all control laws in the form of ordinary differential equations in time, with control torque magnitudes or their equivalent as dependent variables.

\section{Definitions and Notations}

Definitions and notational conventions are as follows (see Fig. 2):

Def. 1. Let $n$ be the number of hinges interconnecting a set of $n+1$ substructures.

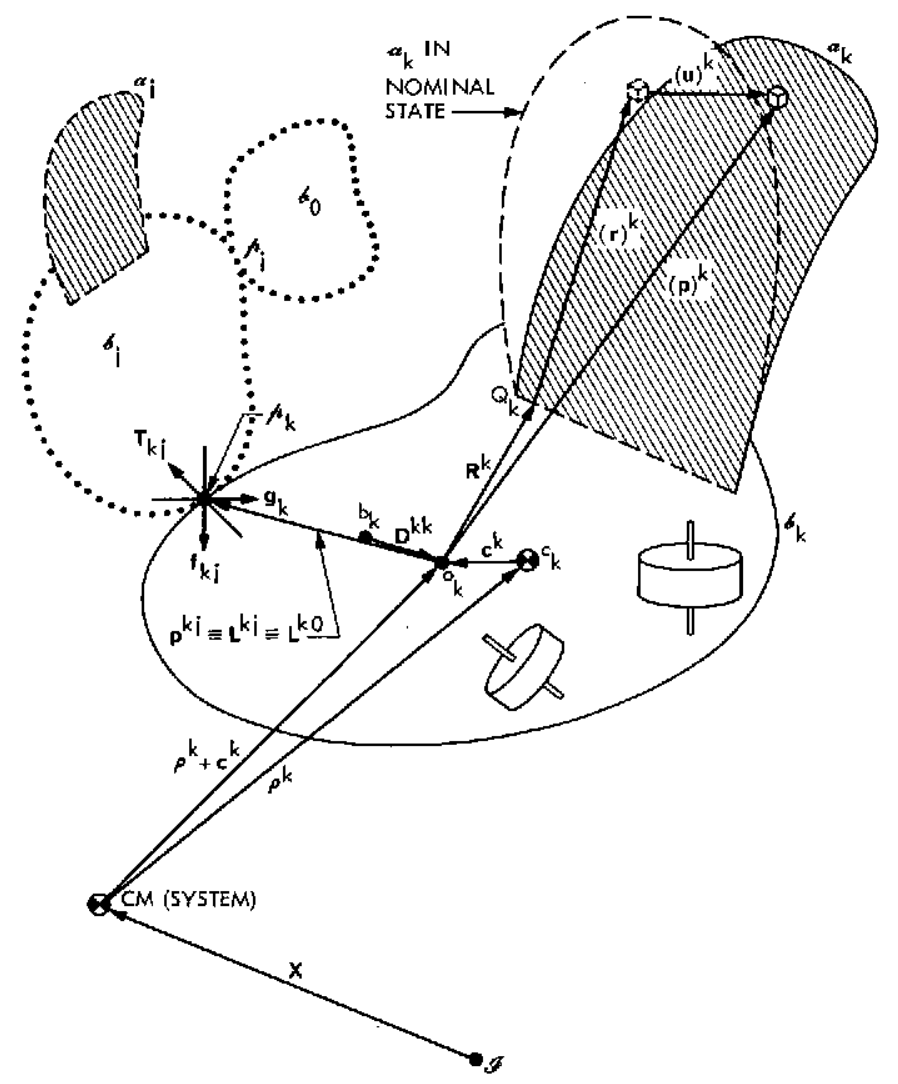

Fig. 2. Definitions for the $k$ th substructure, with $l<k$ 
Def. 2. Define the integer set $B \triangleq\{0,1, \cdots, n\}$.

Def. 3. Define the integer set $\mathscr{P} \triangleq\{1, \cdots, n\}$.

Def.4. Let $b_{0}$ be a label assigned to one rigid body chosen arbitrarily as a reference body, and let $b_{1}, \cdots, b_{n}$ be labels assigned to the rest of the rigid bodies in such a way that if $b_{j}$ is located between $b_{0}$ and $b_{k}$ then $0<j<k$.

Def.5. Define dextral, orthogonal sets of unit vectors $\mathbf{b}_{1}^{k}, \mathbf{b}_{2}^{k}, \mathbf{b}_{3}^{k}$ so as to be imbedded in $\mathscr{G}_{k}$ for $k \in B$, and such that in some arbitrarily selected nominal configuration of the total system, $b_{\alpha}^{k}=b_{\alpha}^{j}$ for $\alpha=1,2,3$ and $k, j \in \mathscr{B}$.

Def. 6. Define

$$
\left\{\mathbf{b}^{k}\right\} \triangleq\left\{\begin{array}{l}
\mathbf{b}_{1}^{k} \\
\mathbf{b}_{2}^{k} \\
\mathbf{b}_{3}^{k}
\end{array}\right\} \quad k \in B
$$

Def. 7. Define $\{\mathbf{i}\}$ as a column array of inertially fixed, dextral, orthogonal unit vectors $\mathbf{i}_{1}, \mathbf{i}_{2}, \mathbf{i}_{3}$.

Def. 8. Let $C$ be the direction cosine matrix defined by

$$
\left\{\mathbf{b}^{0}\right\}=C\{\mathbf{i}\}
$$

Def. 9. Let $\omega^{0} \triangleq\left\{b^{0}\right\}^{T} \omega^{0}$ be the inertial angular velocity vector of $b_{0}$, so that $\omega^{0}$ is the corresponding $3 \times 1$ matrix in basis $\left\{b^{0}\right\}$.

Def. 10. Let $c_{k}$ be the mass center of the $k$ th substructure, $k \in \mathscr{B}$.

Def. 11. Let $h_{k}$ be a point on the hinge axis common to $b_{k}$ and $b_{j}$ for $j<k$ and $k \in \mathscr{P}$.

Def. 12. Let $\mathrm{p}^{k f}$ be the position vector of the hinge point connecting $b_{j}$ and $b_{k}$ from the point $o_{k}$ occupied by $c_{k}$ when the $k$ th substructure is in its nominal state.

Def. 13. Let $\mathrm{c}^{k}$ be the position vector from $c_{k}$ to $o_{k}$.

Def. 14. Let $\boldsymbol{\rho}^{k}$ be the position vector to $c_{k}$ from the system mass center CM.

Def. 15. Let $\mathbf{X}$ be the position vector to CM from an inertially fixed point $\mathscr{I}$, and let $\mathbf{X}=\mathbf{X} \cdot\{\mathbf{i}\}$.

Def. 16. Let $m_{k}$ be the mass of the $k$ th substructure, for $k \in \mathscr{B}$.

Def. 17. Let $(\mathbf{p})^{k}$ be a generic position vector from $o_{k}$ to any point in the $k$ th substructure.

Def. 18. Let $Q_{k}$ be a point common to rigid body $k_{k}$ and flexible appendage $\boldsymbol{a}_{k}$.

Def. 19. Let $\mathbf{R}^{k}=\left\{\mathbf{b}^{k}\right\}^{T} R^{k}$ be the position vector fixed in $k_{k}$ locating $Q_{k}$ with respect to $o_{k}$.

Def. 20. Let $(\mathbf{r})^{k}=\left\{\mathbf{b}^{k}\right\}^{r}(r)^{k}$ be a generic symbol such that $\mathbf{R}^{k}+(\mathbf{r})^{k}$ locates a typical field point in $a_{k}$ with respect to $o_{k}$ when the flexible appendage is in some nominal state (perhaps undeformed). For a discretized appendage $a_{k}$, let $\left(\mathbf{r}^{s}\right)^{k}=\left\{\mathbf{b}^{k}\right\}^{x}\left(\boldsymbol{r}^{s}\right)^{k}$ locate the sth node in the nominal state. 
Def. 21. Define the generic deformation vector $(u)^{k}$ in such a way that ${ }^{2}$

and

$$
(\mathbf{p})^{k} \triangleq \mathbf{R}^{k}+(\mathbf{r})^{k}+(\mathbf{u})^{k}
$$

$$
(p)^{k}=R^{k}+(r)^{k}+(u)^{k}
$$

For a discretized appendage $\boldsymbol{a}_{k}$, let $\left(\mathbf{u}^{s}\right)^{k}=\left\{\mathbf{b}^{k}\right\}^{T}\left(\boldsymbol{u}^{s}\right)^{k}$ be the deformation vector for node $s$.

Def. 22. Let $\mathrm{g}^{k} \stackrel{\Delta}{=}\left\{\mathbf{b}^{k}\right\}^{T} g^{k}$ be a unit vector parallel to the hinge axis through $/ \mu_{k}$.

Def. 23. For $k \in \mathscr{P}$, let $\gamma_{k}$ be the angle of a $g^{\mathrm{k}}$ rotation of $6_{k}$ with respect to the body attached at $p_{k}$. Let $\gamma_{k}$ be zero when $\mathbf{b}_{\alpha}^{k}=\mathbf{b}_{\alpha}^{j}(\alpha=1,2,3 ; j, k \in \mathscr{B})$.

Def. 24. Let $\mathbf{J}^{k} \triangleq\left\{\mathbf{b}^{k}\right\}^{g} J^{k}\left\{\mathbf{b}^{k}\right\}$ be the inertia dyadic of the $k$ th substructure for $o_{k}$, so that $\mathbf{J}^{k}$ is time-variable by virtue of deformations.

Def. 25. Let $\mathbf{F}^{k} \triangleq\left\{\mathbf{b}^{k}\right\}^{r} F^{k}$ be the resultant vector of all forces applied to the $k$ th substructure except for those due to interbody forces transmitted at hinge connections.

Def. 26. Let $\mathbf{T}^{k} \triangleq\left\{\mathbf{b}^{k}\right\}^{x} T^{k}$ be the resultant moment vector with respect to $c_{k}$ of all forces applied to the $k$ th substructure except for those due to interbody forces transmitted at hinge connections.

Def. 27. Let $\tau_{k}$ be the scalar magnitude of the torque component applied to $k_{k}$ in the direction of $\mathrm{g}^{k}$ by the body attached at $\mu_{k}$.

Def. 28. Let $\mathbf{F} \triangleq \sum_{k \in \mathscr{B}} \mathbf{F}^{k}=\left\{\mathbf{b}^{0}\right\}^{r} F$ be the external force resultant for the total system.

Def. 29. Define the scalar $\varepsilon_{s k}$ such that for $k \in \mathscr{B}$ and $s \in \mathscr{P}$

$$
\varepsilon_{g k} \triangleq\left\{\begin{array}{l}
1 \text { if } h_{s} \text { lies between } b_{0} \text { and } b_{k} \\
0 \text { otherwise }
\end{array}\right.
$$

(The $n(n+1)$ scalars $\varepsilon_{s k}$ are called path elements.)

Def. 30. Define $m \triangleq \sum_{k \in \mathbb{B}} m_{k}$, the total system mass.

Def. 31. Let $C^{r j}$ be the direction cosine matrix defined by $\left\{\mathbf{b}^{r}\right\}=C^{r j}\left\{\mathbf{b}^{j}\right\}, r, j \in \mathscr{B}$. (Note that in the nominal state, $C^{r j}=U$, the unit matrix.)

Def. 32. Let $N_{k r}$ denote the index of the body attached to $b_{k}$ and on the path leading to $b_{r}$, and let $N_{k k} \triangleq k$. (These are the network elements.) For notational simplicity, use $N_{k}$ for $N_{k 0}$.

Def. 33. For ${ }^{3} \in \in B-k$, let $\mathbf{L}^{k r} \triangleq \mathbf{p}^{k k_{k r}}$, and let $\mathbf{L}^{k k} \triangleq 0$.

Def. 34. Define $\mathbf{D}^{k k} \triangleq-\Sigma_{i \in \mathbb{B}} \mathbf{L}^{k j} m_{j} / m$ for $k \in \mathscr{B}$.

Def. 35. Let $b_{k}$ be a point fixed in $b_{k}$ such that $\mathbf{D}^{k k}$ is the position vector of $o_{k}$ with respect to $b_{k}$. (This point $b_{k}$ is called the barycenter of the $k$ th substructure in the nominal state.)

\footnotetext{
${ }^{2}$ Superscripts on generic symbols such as $\mathbf{p}, \mathbf{r}$, and $\mathbf{u}$ will be omitted when obvious, as when the symbol appears within an integrand of a definite integral.

${ }^{3}$ For notational brevity, the set $\mathscr{B}-\{k\}$ is designated $\mathscr{B}-k$.
} 
Def. 36. Define $\left\{\mathbf{b}^{k}\right\}^{T} \mathbf{D}^{k j} \triangleq \mathbf{D}^{k j} \stackrel{\Delta}{=} \mathbf{D}^{k k}+\mathbf{L}^{k j}$ for $k, j \in \not B$.

Def. 37. Define the dyadic

$$
\mathbf{K}^{k} \triangleq \sum_{r \in \mathbb{B}} m_{r}\left(\mathbf{D}^{k r} \cdot \mathbf{D}^{k r} \mathbf{U}-\mathbf{D}^{k r} \mathbf{D}^{k r}\right)
$$

where $\mathbf{U}$ is the unit dyadic, and define the corresponding matrix $K^{k} \triangleq$ $\left\{\mathbf{b}^{k}\right\} \cdot \mathbf{K}^{k} \cdot\left\{\mathbf{b}^{k}\right\}^{T}$.

Def. 38. Define

Def. 39. Define

$$
\boldsymbol{\Phi}^{k k} \triangleq \mathbf{K}^{k}+\mathbf{J}^{k} \quad \text { and } \quad \Phi^{k k} \triangleq\left\{\mathbf{b}^{k}\right\} \cdot \boldsymbol{\Phi}^{k k} \cdot\left\{\mathbf{b}^{k}\right\}^{T}
$$

with

$$
\boldsymbol{\Phi}^{k j} \triangleq-m\left(\mathbf{D}^{j k} \cdot \mathbf{D}^{k j} \mathbf{U}-\mathbf{D}^{i k} \mathbf{D}^{k j}\right)
$$

$$
\left\{\mathbf{b}^{j}\right\} \cdot \Phi^{k j} \cdot\left\{\mathbf{b}^{k}\right\}^{r}=-m\left(C^{j k} D^{j k r} C^{j k} D^{k j}-D^{j k} D^{k j T}\right)
$$

Def. 40 . Let the system of forces applied to $b_{k}$ by the attached body $b_{j}$ be equivalent to a resultant force $f^{i j}$ passing through the labeled point $\left(k_{i}\right.$ or $\left./ \beta_{k}\right)$ common to $b_{k}$ and $b_{j}$, plus a torque $\mathbf{T}^{k j}$.

Def. 41. Let $\mathrm{t}^{k j}$ be the kinematical constraint torque applied to $b_{k}$ by $b_{j}$, in such a way that, with Defs. 40,22 , and 27 ,

where

$$
\mathbf{T}^{k j}=\mathbf{t}^{k j}+\delta_{j N_{k}} \tau_{k} \mathbf{g}^{k}-\delta_{k N_{f}} \tau_{j} \mathbf{g}^{j}
$$

$$
\delta_{i j}=1 \text { and } \delta_{j l}=0 \text { for } \ell \neq j .
$$

Def. 42. Let $\omega^{k}=\left\{\mathbf{b}^{k}\right\}^{T} \omega^{k}$ be the inertial angular velocity of $\mathscr{G}_{k}$.

Def. 43. Let $\mathscr{B}_{r}$ be the $r$ th neighbor set for $r \epsilon \mathscr{B}$, such that $k \epsilon \mathscr{B}$, if $\mathscr{k}_{k}$ is attached to $b_{r}$.

Def. 44. Let $\mathscr{B}_{j k}$ be the branch set of integers $r$ such that $r \epsilon \mathscr{B}_{j k}$ if $k=N_{j r}$. Thus $B_{j k}$ consists of the indices of those bodies attached to $\mathscr{b}_{j}$ on a branch which begins with $b_{k}$.

Def. 45. Let $\mathbf{h}^{k}$ be the contribution of rotors in $b_{k}$ to the angular momentum of the $k$ th substructure relative to $b_{k}$ with respect to $o_{k}$, and let $h^{k} \triangleq \mathbf{h}^{k} \cdot\left\{\mathbf{b}^{k}\right\}$.

\section{Derivations of Vector-Dyadic Equations}

In terms of the indicated definitions, Eq. (1) provides, for the $r$ th substructure $(r \in \mathcal{B})$,

$$
\mathbf{F}^{r}+\sum_{s \in \mathbb{B}} \mathbf{f}^{r s}-m_{r}\left(\ddot{\mathbf{X}}+\ddot{\mathbf{p}}^{r}\right)=\mathbf{0}
$$

and, for the $k$ th substructure $(k \in \notin)$,

$$
\mathbf{T}^{k}+\sum_{i \in \mathscr{B}_{k}} \mathbf{T}^{k j}+\sum_{i \in B_{k}}\left(\mathbf{p}^{k j}+\mathbf{c}^{k}\right) \times \mathbf{f}^{k j}-\dot{\mathbf{H}}^{k}=0
$$

Here a dot over a vector implies time differentiation in an inertial frame of reference. 
As shown in Ref. 5, pp. 32-33, the $k$ th substructure angular momentum $\mathbf{H}^{k}$ can be expanded from basic definitions as

$$
\mathbf{H}^{k} \stackrel{\Delta}{=} \int_{\Delta_{k}}(\mathbf{c}+\mathbf{p}) \times(\dot{\mathbf{c}}+\dot{\mathbf{p}}) d m=\mathbf{J}^{k} \cdot \boldsymbol{\omega}^{k}+\left(m_{k} \dot{\mathbf{c}}^{k} \times \mathbf{c}^{k}+\int_{\sigma_{k}} \mathbf{p} \times \stackrel{\circ}{\mathbf{p}} d m\right.
$$

where superscripts $k$ are dropped from $\mathbf{c}$ and $\mathbf{p}$ within integrals ranging over the substructure $a_{k}$. Here the open circle over a vector implies time differentiation in the reference frame established by $b_{k}$. The contribution of a rotor to the integral last appearing in Eq. (4) may be recognized as a vector fixed in $b_{k}$ and parallel to the rotor axis, with a magnitude equal to the product of rotor spin axis inertia and the relative angular speed of the rotor relative to $b_{k}$; the vector sum of the contributions to this integral of all the rotors in $b_{k}$ is here designated $\mathbf{h}^{k}$. Since $\mathbf{p}^{k}=0$ for any field point in $b_{k}$, Eq. (4) can be written

$$
\mathbf{H}^{k}=\mathbf{J}^{k} \cdot \boldsymbol{\omega}^{k}+m \dot{c}_{k} \dot{\mathbf{c}}^{k} \times \mathbf{c}^{k}+\mathbf{h}^{k}+\int_{\alpha_{k}} \mathbf{p} \times \stackrel{\circ}{\mathbf{p}} d m
$$

limiting the range of integration to the appendage $\boldsymbol{a}_{k}$. Substitution of the inertial time derivative of Eq. (5) into $\mathrm{Eq}$. (3) produces, for $k \in \ell$,

$$
\begin{array}{r}
\mathbf{T}^{k}+\sum_{j \in \mathscr{Q}_{k}} \mathbf{T}^{k j}+\sum_{j \in \mathscr{B}_{k}} \mathbf{p}^{k j} \times \mathbf{f}^{k j}+\mathbf{c}^{k} \times \sum_{j \in \mathscr{B}_{k}} \mathbf{f}^{k j}-\frac{i d}{d t}\left(\mathbf{J}^{k} \cdot \boldsymbol{\omega}^{k}\right)-m_{k} \ddot{\mathbf{c}}^{k} \times \mathbf{c}^{k}-\dot{\mathbf{h}}^{k} \\
-\frac{i d}{d t} \int_{\alpha_{k}} \mathbf{p} \times \stackrel{\circ}{\mathbf{p}} d m=0
\end{array}
$$

where either an overdot or the presuperscript $i$ denotes an inertial reference frame for time differentiation of a vector.

The integral in Eq. (6) cannot be evaluated explicitly without adopting a specific mathematical model of the flexible appendage; nor can one go beyond the integral representation of the substructure inertia dyadic $J^{k}$ and the vector $\mathbf{c}^{k}$, which vector establishes the shift of the substructure mass center $c_{k}$ relative to body-fixed point $o_{k}$ due to appendage deformation. Without commitment to a particular appendage model, one can accept (dropping subscripts within integrands)

$$
\mathbf{J} \triangleq \int_{\Delta_{k}}(\mathbf{p} \cdot \mathbf{p} \mathbf{U}-\mathbf{p} \mathbf{p}) d m
$$

and

$$
\mathbf{c}^{k} \stackrel{\Delta}{=}-\frac{1}{m_{k}} \int_{n_{k}} \mathbf{u} d m
$$

If the deformations $\mathbf{u}$ are to be assumed "small," the term $-m_{k} \ddot{\mathbf{c}}^{k} \times \mathbf{c}^{k}$ in Eq. (6) becomes negligible and can be ignored.

Since it is possible to manipulate Eqs. (2) and (6) to eliminate redundant variables and kinematic constraint forces and torques without making any restrictive assumptions concerning the appendage model or the size of its deformations, no 
inhibiting assumptions will be imposed until specific cases are considered at the conclusion of this section.

The immediate objective is the extraction from the $6 n+6$ equations given by Eqs. (2) and (6) of $6+n$ equations in the $6+n$ unknowns established by the vectors $\mathbf{X}$ and $\omega^{0}$ and the scalars $\gamma_{1}, \cdots, \gamma_{n}$ which define the relative rotations of contiguous rigid bodies. We must expect these equations also to involve unknown deformation variables for the appendages and rate variables for the rotors, but we must eliminate all interaction forces (typified by $\mathbf{f}^{k j}$ ) and all interaction torques (typified by $\mathbf{T}^{k j}$ ) except for those having the direction of the corresponding hinge axes, since these hinge-axis torques are known functions of hinge rotation or some more general control law. We must also eliminate the unknown position vectors typified by $\boldsymbol{\rho}^{r}$, replacing them by explicit functions of the hinge angles $\gamma_{1}, \cdots, \gamma_{n}$ and the system geometry, and eliminate all $\omega^{k}$ for $k \in \mathscr{P}$ in favor of terms involving $\omega^{0}$ and $\dot{\gamma}_{r}$ for $r \in \Theta$.

Simply by summing all equations defined by Eq. (2), we obtain the familiar result

$$
\mathbf{F}=m \ddot{\mathbf{x}}
$$

where $M$ is the total system mass and $\mathbf{F}$ is the resultant of all external forces $\mathbf{F}^{r}$ for $r \in B$. Substituting from Eq. (9) back into Eq. (2) and rearranging produces

$$
\mathbf{F}^{r}+\sum_{s \in \mathbb{B}_{r}} \mathbf{f}^{r s}-m_{r}\left(\mathbf{F} / m+\ddot{\rho}^{r}\right)=0
$$

or, for $r \in \mathscr{B}$,

$$
\sum_{s \in \mathscr{S} \mathfrak{B}_{r}} \mathbf{f}^{r s}=-\mathbf{F}^{r}+m_{r}\left(\mathbf{F} / m+\ddot{\mathbf{p}}^{r}\right)
$$

Summing over the branch set $\mathscr{B}_{k j}$ (see Def. 44) provides

$$
\mathbf{f}^{j k}=\sum_{r \in B_{k+1}} \sum_{s \in B_{r}} \mathbf{f}^{r s}=-\sum_{r \in \mathcal{B}_{k_{j}}}\left[\mathbf{F}^{r}-m_{r}\left(\mathbf{F} / M n+\ddot{\boldsymbol{\rho}}^{r}\right)\right]
$$

so that, by Newton's third law,

$$
\mathbf{f}^{k j}=-\mathbf{f}^{j k}=\sum_{r \in B_{k j}}\left[\mathbf{F}^{r}-m_{r}\left(\mathbf{F} / m+\ddot{\mathbf{p}}^{r}\right)\right]
$$

Substituting Eqs. (10) and (11) into Eq. (6) eliminates all interaction forces from the rotational equations, providing

$$
\begin{aligned}
& \mathbf{T}^{k}+\sum_{i \in B_{k}} \mathbf{T}^{k j}+\sum_{j \in Q_{k}} \mathbf{p}^{k j} \times \sum_{r \in B_{k}}\left[\mathbf{F}^{r}-m_{r}\left(\mathbf{F} / m+\ddot{\boldsymbol{\rho}}^{r}\right)\right] \\
& +\mathbf{c}^{k} \times\left[-\mathbf{F}^{k}+m_{k}\left(\mathbf{F} / m+\ddot{\mathbf{p}}^{k}\right)\right]-\frac{{ }^{i} d}{d t}\left(\boldsymbol{J}^{k} \cdot \boldsymbol{\omega}^{k}\right)-m_{k} \ddot{\mathbf{c}}^{k} \times \mathbf{c}^{k} \\
& -\dot{\mathbf{h}}^{k}-\frac{{ }^{i} d}{d t} \int_{a_{k}} \mathbf{p} \times \stackrel{\circ}{\mathbf{p}} d m=0 \quad(k \in \mathscr{B})
\end{aligned}
$$


Equation (12) simplifies when written in terms of the vectors found in Defs. 33-36 in the preceding section, due to the identity, for $k \in \mathscr{B}$,

$$
\begin{aligned}
& \sum_{i \in \Omega_{k}} \mathbf{p}^{k j} \times \sum_{r \in \Theta_{k i}}\left[\mathbf{F}^{r}-m_{r}\left(\mathbf{F} / m+\ddot{\boldsymbol{\rho}}^{r}\right)\right]=\sum_{r \in \mathbb{B}} \mathbf{L}^{k r} \times\left[\mathbf{F}^{r}-m_{r}\left(\mathbf{F} / m+\ddot{\boldsymbol{\rho}}^{r}\right)\right] \\
& =\sum_{r \in \mathcal{B}} \mathbf{L}^{k r} \times \mathbf{F}^{r}+\mathbf{D}^{k k} \times \mathbf{F}-\sum_{r \in \mathcal{B}} \mathbf{L}^{k r} \times m_{r} \ddot{\mathbf{\rho}}^{r} \\
& =\sum_{r \in \mathscr{B}}\left(\mathbf{L}^{k r}+\mathbf{D}^{k k}\right) \times \mathbf{F}^{r}-\sum_{r \in \mathscr{B}} \mathbf{L}^{k r} \times m_{r} \ddot{\mathbf{p}}^{r} \\
& =\sum_{r \in \mathscr{B}}\left(\mathbf{D}^{k r} \times \mathbf{F}^{r}-\mathbf{L}^{k r} \times\left(m_{\tau} \ddot{\mathbf{P}}^{r}\right)\right.
\end{aligned}
$$

Substituting Eq. (13) into Eq. (12) simplifies its appearance somewhat, but there remains the problem of eliminating the unknown kinematic constraint torques which are present within the interaction torques typified by $\mathbf{T}^{k j}$. By summing over all $n+1$ vector equations defined by Eq. (12), we can, by virtue of Newton's third law, obtain one vector equation involving no interaction torques at all; this summation gives

$$
\begin{array}{r}
\sum_{\mathbf{k} \in \mathbb{B}}\left\{\mathbf{T}^{k}+\sum_{r \in \mathcal{B}}\left(\mathbf{D}^{k r} \times \mathbf{F}^{r}-\mathbf{L}^{k r} \times m_{r} \ddot{\mathbf{\rho}}^{r}\right)+\mathbf{c}^{k} \times\left[m_{k}\left(\ddot{\mathbf{\rho}}^{k}+\mathbf{F} / m\right)-\mathbf{F}^{k}\right]\right. \\
\left.-\frac{{ }^{i} d}{d t}\left(\mathbf{J}^{k} \cdot \boldsymbol{\omega}^{k}\right)-m_{k} \ddot{\mathbf{c}}^{k} \times \mathbf{c}^{k}-\dot{\mathbf{h}}^{k}-\frac{{ }^{i} d}{d t} \int_{\pi_{k}} \mathbf{p} \times \stackrel{\circ}{\mathbf{p}} d m\right\}=0
\end{array}
$$

Equation (14), like Eq. (9), is free of kinematic constraint forces and torques, so that these two vector equations can be preserved in the final set that is our objective. The remaining $n$ scalar equations can be obtained by summing equations obtained from Eq. (12) over branch sets in order to isolate interaction torques such as $\mathbf{T}^{k j}$, and then by dot-multiplying each expression by the unit vector parallel to the corresponding hinge axis. In particular, if we introduce the network elements found in Def. 32 of the previous section, we can sum over the branch set $\mathfrak{B}_{N_{s} s}$ and get, for $s \in \mathbb{B}$,

$$
\begin{aligned}
& \mathbf{T}^{s N_{s}}+\sum_{k \in \mathscr{B} \boldsymbol{s}_{s}}\left\{\mathbf{T}^{k}+\sum_{r \in \mathcal{B}}\left(\mathbf{D}^{k r} \times \mathbf{F}^{r}-\mathbf{L}^{k r} \times m_{+} \ddot{\mathrm{P}}^{r}\right)\right. \\
& +\mathbf{c}^{k} \times\left[m_{k}\left(\ddot{\mathbf{P}}^{k}+\mathbf{F} / m\right)-\mathbf{F}^{k}\right]-\frac{i}{d t}\left(\mathbf{J}^{k} \cdot \omega^{k}\right)-m_{k} \ddot{\mathbf{c}}^{k} \times \mathbf{c}^{k}-\dot{\mathbf{h}}^{k} \\
& \left.-\frac{i d}{d t} \int_{a_{\mathrm{k}}} \mathbf{p} \times \stackrel{\circ}{\mathbf{p}} d m\right\}=0
\end{aligned}
$$

By Def. $32, N_{s}<s$, so that the labeled point on the hinge axis common to $b_{s}$ and $b_{N_{s}}$ is, by Def. 11 , called $\ell_{s}$; the unit vector parallel to the hinge axis is, by Def. 22 , called $\mathbf{g}^{s}$; and the interaction torque component of $\mathbf{T}^{s{ }^{*}}{ }_{\mathrm{v}}$ parallel to $\mathbf{g}^{s}$ is, by Def. 27 , 


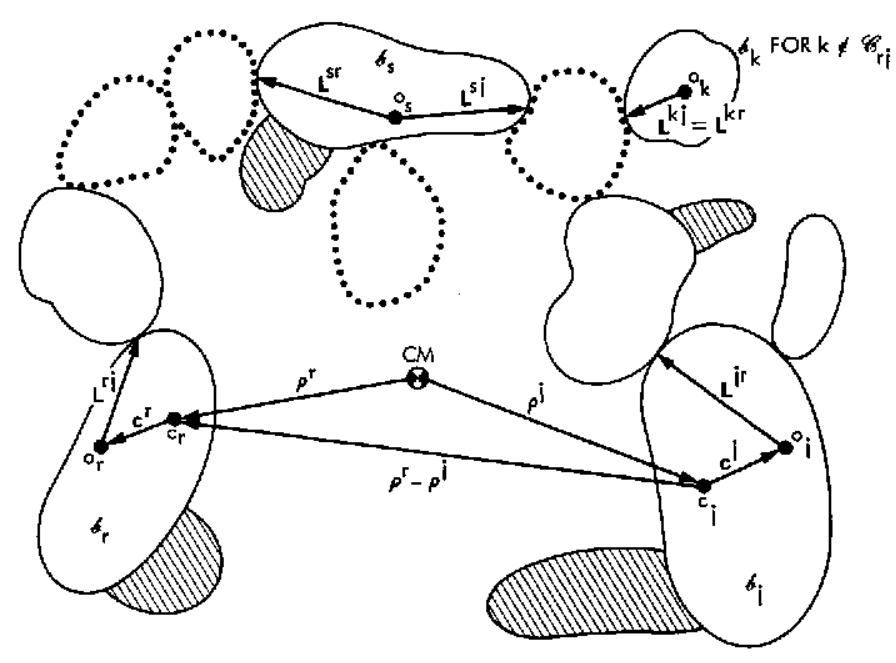

Fig. 3. System geometry

called $\tau_{k}$. Hence the dot product of Eq. (15) with $\mathrm{g}^{8}$ provides the scalar equation, for $s \in \mathscr{B}$,

$$
\begin{aligned}
\tau_{s}+\mathbf{g}^{8} \cdot \sum_{k \in B B_{s}}\left\{\mathbf{T}^{k}+\sum_{r \in \mathcal{B}}\left(\mathbf{D}^{k r} \times \mathbf{F}^{r}-\mathbf{L}^{k r} \times m_{r} \ddot{\mathbf{\rho}}^{r}\right)+\mathbf{c}^{k} \times\left[m_{k}\left(\ddot{\mathbf{\rho}}^{k}+\mathbf{F} / m\right)-\mathbf{F}^{k}\right]\right. \\
\left.-\frac{{ }^{i} d}{d t}\left(\mathbf{J}^{k} \cdot \boldsymbol{\omega}^{k}\right)-O m_{k} \ddot{\mathbf{c}}^{k} \times \mathbf{c}^{k}-\dot{\mathbf{h}}^{k}-\frac{{ }^{i} d}{d t} \int_{\boldsymbol{a}_{k}} \mathbf{p} \times \stackrel{\circ}{\mathbf{p}} d m\right\}=0
\end{aligned}
$$

Equations (9), (14), and (16) provide the required $6+n$ scalar equations free of kinematic constraint forces and torques, but they are not yet in final vector-dyadic form. The variable vectors typified by $\rho^{r}$ must first be expressed in terms of the system geometry, the deformation variables, and a subset of the $6+n$ scalars required to define $\mathrm{X}, \omega^{0}$, and the $n$ angles of relative rotation at the $n$ hinges. As a first step in this direction, we define $C_{r j}$ as the set of indices of bodies lying on the direct path between $b_{r}$ and $b_{j}$, and then (referring to Fig. 3) we can write

$$
\boldsymbol{\rho}^{r}-\mathbf{\rho}^{j}=\mathbf{c}^{j}+\mathbf{L}^{j r}+\sum_{s \in e_{r+1}}\left(\mathbf{L}^{s r}-\mathbf{L}^{s i}\right)-\mathbf{L}^{r i}-\mathbf{c}^{r}
$$

But $\mathbf{L}^{i j}=\mathbf{L}^{r r}=0$, by Def. 33, and for any index $s$ in the set $\measuredangle B-\bigodot_{r j}$ the sum $\mathbf{L}^{s r}-\mathbf{L}^{s j}=0$, so that Eq. (18) can be written more simply as

$$
\boldsymbol{\rho}^{r}-\boldsymbol{\rho}^{j}=\sum_{s e \mathcal{B}}\left(\mathbf{L}^{8 r}-\mathbf{L}^{s f}\right)+\mathbf{c}^{j}-\mathbf{c}^{r}
$$

Multiplying Eq. (18) by $m_{j} /(m$ and summing over all $j \in \mathscr{B}$ produces

$$
\sum_{i \in \mathscr{B}} \frac{m_{j}}{m} \boldsymbol{\rho}^{r}-\sum_{j \in \mathscr{B}} \frac{m_{j}}{m} \boldsymbol{\rho}^{i}=\sum_{i \in \mathscr{B}} \sum_{\boldsymbol{s} \in \mathcal{B}} \frac{m_{j}}{m}\left(\mathbf{L}^{s r}-\mathbf{L}^{s j}\right)+\sum_{i \in \mathscr{O}} \frac{m_{j}}{m}\left(\mathbf{c}^{j}-\mathbf{c}^{r}\right)
$$

But

$$
\sum_{j \in \mathfrak{B}} m_{j} \mathrm{p}^{j}=0
$$


by definition of $\mathrm{CM}$, and

$$
\sum_{i \in B} m_{i} \triangleq m
$$

so we have

$$
\boldsymbol{\rho}^{r}=\sum_{i \in \mathcal{B}} \sum_{s \in \mathcal{B}} \frac{m_{j}}{m}\left(\mathbf{L}^{s r}-\mathbf{L}^{s j}\right)+\frac{1}{m} \sum_{i \in \mathbb{B}} m_{j} \mathbf{c}^{j}-\mathbf{c}^{r}
$$

Reversing the summation sequence in the first term, changing the index symbol in the final summation, and substituting the vectors introduced in Defs. 34 and 36, we find simply that, for $r \in \mathscr{B}$,

$$
\boldsymbol{\rho}^{r}=\sum_{s \in \mathbb{B}}\left(\mathbf{L}^{s r}+\mathbf{D}^{s s}+\frac{m_{s}}{m} \mathbf{c}^{s}\right)-\mathbf{c}^{r}=\sum_{s \in \mathfrak{B}}\left(\mathbf{D}^{s r}+\frac{m_{s}}{m} \mathbf{c}^{s}\right)-\mathbf{c}^{r}
$$

Equations (14) and (16) contain not $\rho^{r}$ itself but combinations which, with Eq. (19), can be written as

$$
\mathbf{c}^{k} \times m_{k} \ddot{\mathrm{p}}^{k}=m_{k} \mathbf{c}^{k} \times\left[\sum_{r \in B}\left(\ddot{\mathbf{D}}^{r k}+\frac{m_{r}}{m} \ddot{\mathbf{c}}^{r}\right)-\ddot{\mathbf{c}}^{k}\right]
$$

and, in terms employing the symbols of Defs. 33-37,

$$
\begin{aligned}
& -\sum_{r \in \mathscr{R}} \mathbf{L}^{k r} \times m_{r} \ddot{\boldsymbol{\rho}}^{r}=-\sum_{r \in \mathcal{B}} \mathbf{L}^{k r} \times m_{r}\left[\sum_{s \in \mathcal{B}}\left(\ddot{\mathbf{D}}^{s r}+\frac{m_{s}}{m} \ddot{\mathbf{c}}^{s}\right)-\ddot{\mathbf{c}}^{r}\right] \\
& =-\sum_{r \in \mathcal{B}} \mathbf{L}^{k r} \times m_{r}\left[\ddot{\mathbf{D}}^{k r}+\sum_{s \in \mathscr{B}-k} \ddot{\boldsymbol{D}}^{s r}+\sum_{s \in \mathfrak{B}} \frac{m_{8}}{m} \ddot{\mathbf{c}}^{s}-\ddot{\mathbf{c}}^{r}\right] \\
& =\sum_{r \in \mathcal{B}}\left(\mathbf{D}^{k k}-\mathbf{D}^{k r}\right) \times\left[m_{r} \ddot{\mathbf{D}}^{k r}+\frac{m_{r}}{m}\left(\sum_{s \in \mathfrak{B}} m_{s} \ddot{\mathbf{c}}^{s}-m \ddot{\ddot{\mathbf{c}}}^{r}\right)\right] \\
& -\sum_{r \in \mathbb{B}-\mathbf{k}} \sum_{s \in \Re-k-r} m_{r} \mathbf{L}^{k r} \times \ddot{\mathbf{D}}^{s r}-\sum_{r \in \mathscr{B}-\mathbf{k}} m_{r} \mathbf{L}^{k r} \times \ddot{\mathbf{D}}^{r r} \\
& =\mathbf{D}^{k k} \times \sum_{r \in \mathcal{B}} m_{r} \ddot{\mathbf{D}}^{k r}-\frac{i d}{d t}\left(\sum_{r \in \mathbb{B}} \mathbf{D}^{k r} \times m_{\boldsymbol{r}} \dot{\mathbf{D}}^{k r}\right)+\mathbf{D}^{k k} \times \sum_{s \in \mathbb{B}} m_{B} \ddot{\mathbf{c}}^{s} \\
& -\mathbf{D}^{k \pi} \times \sum_{r \in \mathscr{B}} m_{r} \ddot{\mathbf{c}}^{r}-\left(\sum_{r \in \mathscr{G}} \mathbf{D}^{k+} m_{r}\right) \times \sum_{s \in \mathscr{A}} \frac{m_{s}}{m} \ddot{\mathbf{c}}^{s} \\
& +\sum_{r \in \mathcal{B}} \mathbf{D}^{k r} \times m_{r} \ddot{\mathbf{c}}^{r}-\sum_{r \in \mathcal{B}-\mathbf{k}} \sum_{s \in \mathcal{B}-k-r} m_{r} \mathbf{L}^{k r} \times \ddot{\mathbf{D}}^{\beta r} \\
& +\sum_{r \in \mathscr{B}-k} \sum_{s \in \mathbb{B}-r} m_{s} \mathbf{L}^{k r} \times \ddot{\mathbf{D}}^{r a} \\
& =0-\frac{i d}{d t}\left[\sum_{r \in \mathcal{B}} m_{5} \mathbf{D}^{k r} \times\left(\boldsymbol{\omega}^{k} \times \mathbf{D}^{k r}\right)\right]+0-0+\sum_{r \in \mathcal{B}} \mathbf{D}^{k r} \times m_{r} \ddot{\mathbf{c}}^{r} \\
& -\sum_{r \in \mathbb{B}-\mathbf{k}} \sum_{s \in \mathbb{B}-k-r} m_{8} \mathbf{L}^{k s} \times \ddot{\mathbf{D}}^{r s}+\sum_{r \in \mathbb{B}-k} \sum_{s \in \mathbb{B}-r} m_{s} \mathbf{L}^{k r} \times \ddot{\mathbf{D}}^{r s}
\end{aligned}
$$




$$
\begin{aligned}
= & -\frac{{ }^{i} d}{d t}\left[\left\{\sum_{r \in \mathscr{B}} m_{r}\left(\mathbf{D}^{k r} \cdot \mathbf{D}^{k r} \mathbf{U}-\mathbf{D}^{k r} \mathbf{D}^{k r}\right)\right\} \cdot \boldsymbol{\omega}^{k}\right] \\
& +\sum_{r \in \mathbb{B}} m_{r} \mathbf{D}^{k r} \times \ddot{\mathbf{c}}^{r}+\sum_{r \in \mathbb{B}-\boldsymbol{k}} \sum_{s \in \mathbb{Q} \rightarrow r} m_{8}\left(\mathbf{L}^{k r}-\mathbf{L}^{k s}\right) \times \ddot{\mathbf{D}}^{r s}
\end{aligned}
$$

or

$$
\begin{aligned}
-\sum_{r \in \mathcal{B}} \mathbf{L}^{k r} \times m_{r} \ddot{\boldsymbol{\rho}}^{r}= & -\frac{{ }^{i} d}{d t}\left[\mathbf{K}^{k} \cdot \boldsymbol{\omega}^{k}\right]+\sum_{r \in \mathscr{B}} m_{r} \mathbf{D}^{k r} \times \ddot{\mathbf{c}}^{r} \\
& +\sum_{r \in \mathcal{B}-\mathbf{k}} \sum_{s \in \mathscr{B}} m_{s}\left(\mathbf{L}^{k r}-\mathbf{L}^{k s}\right) \times \ddot{\mathbf{D}}^{r s}
\end{aligned}
$$

In the development lcading to Eq. (21), it was rccognized that

$$
\sum_{s \in \mathscr{B}} m_{s} \mathbf{D}^{r s}=0
$$

(reflecting the significance of the barycenter as the mass center of the undeformed augmented body, which consists of the substructure augmented at each connection point by a particle having the mass of the corresponding branch set). It was also noted that

$$
\begin{aligned}
-\sum_{r \in \mathbb{B}-k} \sum_{s \in \mathbb{R}-k-r} m_{r} \mathbf{L}^{k r} \times \ddot{\mathbf{D}}^{s r} & =-\sum_{s \in \mathcal{B}-\mathbf{k}} \sum_{r \in \mathscr{B}-k-s} m_{r} \mathbf{L}^{k r} \times \ddot{\mathbf{D}}^{s r} \\
& =-\sum_{r \in \mathscr{B}-\mathbf{k}} \sum_{s \in \mathscr{B}-k-r} m_{s} \mathbf{L}^{k s} \times \ddot{\mathbf{D}}^{r s}
\end{aligned}
$$

where the second step involves simply relabeling indices.

Finally, we can recognize in Eq. (21) that the quantity $\mathbf{L}^{k r}-\mathbf{L}^{k s}$ is zero for any index $s$ corresponding to a body which lies anywhere on the branch which begins with $\mathscr{b}_{N_{k r}}$ and includes $b_{r}$, and for any other index the quantity $\mathbf{D}^{r s}$ is also $\mathbf{D}^{r k}$. Thus, in Eq. (21), $\ddot{\mathbf{D}}^{r s}$ can be replaced by $\ddot{\mathbf{D}}^{r k}$, with the consequence

$$
\begin{aligned}
-\sum_{r \in \mathcal{B}} \mathbf{L}^{k r} \times m_{r} \ddot{\boldsymbol{\rho}}^{r}= & -\frac{{ }^{i} d}{d t}\left(\mathbf{K}^{k} \cdot \boldsymbol{\omega}^{k}\right)+\sum_{r \in \mathcal{B}} m_{r} \mathbf{D}^{k r} \times \ddot{\mathbf{c}}^{r} \\
& +\sum_{r \in \mathcal{B}-\mathbf{k}} \sum_{\boldsymbol{s} \in \mathcal{B}} m_{s}\left(\mathbf{L}^{k r}-\mathbf{L}^{k s}\right) \times \ddot{\mathbf{D}}^{r k} \\
= & -\frac{{ }^{i} d}{d t}\left(\boldsymbol{K}^{k} \cdot \boldsymbol{\omega}^{k}\right)+\sum_{r \in \mathcal{B}} m_{r} \mathbf{D}^{k r} \times \ddot{\mathbf{c}}^{r} \\
& +\sum_{r \in \mathcal{B}-\mathbf{k}}\left[\left(m \mathbf{L}^{k r}-\sum_{s \in \mathcal{B}} m_{s} \mathbf{L}^{k s}\right) \times \ddot{\mathbf{D}}^{r k}\right]
\end{aligned}
$$

or, with Defs. 34 and 36,

$$
-\sum_{r \in \Omega \mathcal{B}} \mathbf{L}^{k r} \times m_{r} \ddot{\boldsymbol{\rho}}^{r}=-\frac{i d}{d t}\left(\mathbf{K}^{k} \cdot \boldsymbol{\omega}^{k}\right)+\sum_{r \in \mathcal{B}} m_{r} \mathbf{D}^{k r} \times \ddot{\mathbf{c}}^{r}+\sum_{r \in \mathcal{B}-k} m \mathbf{D}^{k r} \times \ddot{\mathbf{D}}^{r k}
$$

By substituting Eqs. (20) and (22) into Eqs. (14) and (16), we can obtain the desired vector-dyadic equations. In this substitution, it becomes apparent that $\mathbf{K}^{k}$ 
and $\mathbf{J}^{k}$ always appear in combination, suggesting the introduction of the new dyadic $\Phi^{k k}$ (see Def. 38). Then the new versions of Eqs. (14) and (16) become

$$
\begin{aligned}
& \sum_{k \in \mathcal{B}}\left\{\mathbf{T}^{k}+\sum_{r \in \mathcal{B}} \mathbf{D}^{k r} \times \mathbf{F}^{r}+\mathbf{c}^{k} \times\left(\frac{m_{k}}{m} \mathbf{F}-\mathbf{F}^{k}\right)+\sum_{r \in \mathcal{B}} m_{r} \mathbf{D}^{k r} \times \ddot{\mathbf{c}}^{r}\right. \\
& +m_{k} \mathbf{c}^{k} \times \sum_{r e \mathscr{B}}\left(\ddot{\mathbf{D}}^{r k}+\frac{m_{r}}{m} \ddot{\mathbf{c}}^{r}\right)-\frac{i d}{d t}\left(\boldsymbol{\Phi}^{k k} \cdot \boldsymbol{\omega}^{k}\right\rangle+\sum_{r \in \mathscr{B}-k} m \mathbf{D}^{k r} \times \ddot{\mathbf{D}}^{+k}-\dot{\mathbf{h}}^{k} \\
& \left.-\frac{{ }^{i} d}{d t} \int_{a_{k}} \mathbf{p} \times \stackrel{\circ}{\mathbf{p}} d m\right\}=0
\end{aligned}
$$

and, with judicious use of the path elements found in Def. 29,

$$
\begin{aligned}
\tau_{s}+\mathbf{g}_{s} \cdot \sum_{k \in \mathcal{P}} \varepsilon_{s k}\left\{\mathbf{T}^{k}+\sum_{r \in \mathbb{Q}} \mathbf{D}^{k r} \times \mathbf{F}^{r}+\mathbf{c}^{k} \times\left(\frac{m_{k}}{m} \mathbf{F}-\mathbf{F}^{k}\right)\right. \\
+\sum_{r \in \mathscr{B}} m_{r} \mathbf{D}^{k r} \times \ddot{\mathbf{c}}^{r}+m_{k} \mathbf{c}^{k} \times \sum_{r \in \mathfrak{B}}\left(\ddot{\mathbf{D}}^{r k}+\frac{m_{r}}{m} \ddot{\mathbf{c}}^{r}\right)-\frac{i d}{d t}\left(\mathbf{\Phi}^{k k} \cdot \omega^{k}\right) \\
\left.+\sum_{r \in \mathscr{Q}-k} m \mathbf{D}^{k r} \times \ddot{\mathbf{D}}^{+k}-\dot{\mathbf{h}}^{k}-\frac{i d}{d t} \int_{a_{k}} \mathbf{p} \times \stackrel{\circ}{\mathbf{p}} d m\right\}=0
\end{aligned}
$$

Equations (23) and (24) can be restated in more useful form by expanding terms involving time derivatives relative to inertial space to obtain time derivatives relative to reference frames established by individual substructures, plus additional undifferentiated terms. In particular, noting that $\mathbf{D}^{r k}$ is fixed in $k_{r}$, we can substitute

$$
\ddot{D}^{r k}=\dot{\omega}^{r} \times \mathbf{D}^{r k}+\boldsymbol{\omega}^{r} \times\left(\boldsymbol{\omega}^{r} \times \mathbf{D}^{r k}\right)
$$

so that in terms of Def. 39 , we have

$$
\sum_{r e \mathscr{B}-\mathbf{k}} m \mathbf{D}^{k^{k}} \times \ddot{\mathbf{D}}^{r k}=-\sum_{r \in \mathbb{B}-\boldsymbol{k}} \boldsymbol{\Phi}^{k r} \cdot \dot{\boldsymbol{\omega}}^{r}+m \sum_{r \in \mathscr{B}-\mathbf{k}} \mathbf{D}^{k r} \times\left[\boldsymbol{\omega}^{r} \times\left(\boldsymbol{\omega}^{r} \times \mathbf{D}^{r k}\right)\right]
$$

The parallel expression for $\ddot{\mathbf{c}}^{r}$ becomes

$$
\ddot{\mathbf{c}}^{r}=\stackrel{\circ \mathrm{c}^{r}}{ }+2 \boldsymbol{\omega}^{r} \times \ddot{\mathbf{c}}^{r}+\dot{\boldsymbol{\omega}}^{r} \times \mathbf{c}^{r}+\omega^{r} \times\left(\omega^{r} \times \mathbf{c}^{r}\right)
$$

with open circles representing time differentiation in the reference frame established by $\mathfrak{b}_{r}$.

Other time differentiations in inertial space expand as

$$
\begin{gathered}
\dot{\mathbf{h}}^{k}=\stackrel{\circ}{\mathbf{h}}^{k}+\boldsymbol{\omega}^{k} \times \mathbf{h}^{k} \\
\frac{{ }^{i} d}{d t}\left(\boldsymbol{\Phi}^{k k} \cdot \boldsymbol{\omega}^{k}\right)=\boldsymbol{\Phi}^{k k} \cdot \dot{\boldsymbol{\omega}}^{k}+\boldsymbol{\omega}^{k} \times \boldsymbol{\Phi}^{k k} \cdot \boldsymbol{\omega}^{k}+\stackrel{\circ}{\boldsymbol{\Phi}}^{k k} \cdot \boldsymbol{\omega}^{k}
\end{gathered}
$$


and

$$
\frac{i d}{d t} \int_{a_{k}} \mathbf{p} \times \stackrel{\circ}{\mathbf{p}} d m=\int_{a_{k}} \mathbf{p} \times \stackrel{\circ}{\mathbf{p}} d m+\omega^{k} \times \int_{a_{k}}(\mathbf{p} \times \stackrel{\circ}{\mathbf{p}}) d m
$$

with the open circle indicating time differentiation in the reference frame established by the local substructure (here $b_{k}$ ).

By combining Eqs. (23-30), we can obtain the vector-dyadic equations of rotation in the form

and

$$
\sum_{k \in \mathscr{B}} \mathbf{W}^{k}=0
$$

where

$$
\tau_{s}+\mathbf{g}^{s} \cdot \sum_{k \in \mathscr{S}} e_{8 k} \mathbf{W}^{k}=0
$$

$$
\begin{aligned}
& \mathbf{W}^{k} \stackrel{\Delta}{=} \mathbf{T}^{k}+\sum_{r \in \mathfrak{B}} \mathbf{D}^{k r} \times \mathbf{F}^{r}+\mathbf{c}^{k} \times\left(\frac{m_{k}}{m} \mathbf{F}-\mathbf{F}^{k}\right) \\
& +\sum_{r \in \mathscr{B}} m_{r} D^{k r} \times\left[\dot{\mathbf{c}}^{r}+2 \omega^{r} \times \stackrel{\circ}{r}^{r}+\dot{\boldsymbol{\omega}}^{r} \times \mathbf{c}^{r}+\omega^{r} \times\left(\omega^{r} \times \mathbf{c}^{r}\right)\right] \\
& +m_{k} \mathbf{c}^{k} \times \sum_{r \in \mathscr{B}}\left[\dot{\omega}^{r} \times \mathbf{D}^{r k}+\boldsymbol{\omega}^{r} \times\left(\boldsymbol{\omega}^{r} \times \mathbf{D}^{r k}\right)\right] \\
& +m_{k} \mathbf{c}^{k} \times \sum_{r \in \mathscr{B}} \frac{m_{r}}{m}\left[\mathbf{c}^{\circ}+2 \boldsymbol{\omega}^{r} \times \stackrel{\circ}{\mathbf{c}}^{r}+\dot{\boldsymbol{\omega}}^{r} \times \mathbf{c}^{r}+\boldsymbol{\omega}^{r} \times\left(\boldsymbol{\omega}^{r} \times \mathbf{c}^{r}\right)\right]-\boldsymbol{\Phi}^{k k} \cdot \dot{\boldsymbol{\omega}}^{k} \\
& -\sum_{r \in \mathbb{B}-k}\left(\boldsymbol{\Phi}^{k r} \cdot \dot{\boldsymbol{\omega}}^{r}\right)+m \sum_{r \in \mathbb{B}-k} \mathbf{D}^{k r} \times\left[\boldsymbol{\omega}^{r} \times\left(\boldsymbol{\omega}^{r} \times \mathbf{D}^{r k}\right)\right]-\boldsymbol{\omega}^{k} \times \boldsymbol{\Phi}^{k k} \cdot \boldsymbol{\omega}^{k} \\
& -\stackrel{\circ}{\mathbf{h}^{k}}-\boldsymbol{\omega}^{k} \times \mathbf{h}^{k}-\stackrel{\circ}{\boldsymbol{\Phi}^{k k} \cdot \boldsymbol{\omega}^{k}}-\int_{\boldsymbol{a}_{\mathrm{k}}} \mathbf{p} \times \stackrel{\circ}{\mathbf{p}} d m-\boldsymbol{\omega}^{k} \times \int_{a_{k}}(\mathbf{p} \times \stackrel{\circ}{\mathbf{p}}) d m
\end{aligned}
$$

To obtain the vector-dyadic equations in their final form, involving the $6+n$ unknowns in $\mathbf{X}, \boldsymbol{\omega}^{0}$, and $\gamma_{1}, \cdots, \gamma_{n}$ as well as the rotor momentum variables and the deformation variables, we must substitute in Eq. (33) the expressions

and

$$
\boldsymbol{\omega}^{k}=\boldsymbol{\omega}^{0}+\sum_{r \mathscr{Q}} \varepsilon_{+\dot{\gamma}} \dot{\gamma}_{r} \mathbf{g}^{r}
$$

$$
\dot{\omega}^{k}=\dot{\omega}^{0}+\sum_{r \in \Theta} \varepsilon_{r k}\left[\ddot{\gamma}_{r} \mathbf{g}^{r}+\omega^{0} \times \dot{\gamma}_{r} \mathbf{g}^{r}+\sum_{s e \Theta} \varepsilon_{s r} \dot{\gamma}_{s} g^{s} \times \mathbf{g}^{r}\right]
$$

where $\varepsilon_{r k}$ are the path elements found in Def. 29, and Defs. 9, 22, and 23 are also employed.

In combination, Eqs. (9) and (31-35) provide the final form of the vector-dyadic equations of vehicle translation and substructure rotation. In addition to these equations, for each rotor in the system an additional scalar unknown is introduced, and one more differential equation is required. If a rigid axisymmetric rotor with spin axis inertia $\mathscr{Z}_{k s}$ spins relative to body $b_{k}$ at the angular rate $\dot{\psi}_{k s}$ about an axis parallel to the unit vector $\mathbf{b}^{k s}$ fixed in $b_{k}$, and if $\tau_{k s}^{R}$ is the magnitude of the $\mathbf{b}^{k s}$ com- 
ponent of the torque applied to the rotor, then the required additional equation of motion is

$$
\tau_{k s s}^{R}=\mathscr{J}_{k s}\left(\ddot{\psi}_{k s}+\mathbf{b}^{k s} \cdot \dot{\boldsymbol{\omega}}^{k}\right)
$$

If there are $N^{k}$ rotors in $b_{k}$, and $N^{k}$ rotors in the entire system, then Eq. (36) applies for $s=1, \cdots, N^{k}$, with $k \in \mathscr{B}$, so that Eq. (36) contributes $N^{k}$ scalar equations. Moreover, the variables in equations typified by Eq. (36) are related to those in Eq. (33) by

$$
\mathbf{h}^{k}=\sum_{s=1}^{N^{k}} \mathscr{J}_{k s} \dot{\psi}_{k s} \mathbf{b}^{k s}
$$

The scalars typified by $\tau_{k s}^{n}$ must of course be given, either explicitly or implicitly in the form of additional differential equations representing control laws. The same is true of $\tau_{s}, \mathbf{T}^{\epsilon}$, and $\mathbf{F}^{r}$ in Eqs. (33) and (32). Once these issues are settled, however, there remains the problem of defining the nonrigid appendages mathematically and establishing their vibrational motion equations. This issue has been postponed until the end of this section, in order that we might consider several possibilities. The relative advantages of alternative appendage models are considered in detail in Ref. 6 and are only briefly summarized here.

If the appendage $\boldsymbol{a}_{k}$ is of extremcly simple configuration (such as a uniform elastic beam), and the nominal angular velocity $\omega_{k}$ of the base to which it is attached is of a special class (such as zero, or parallel or orthogonal to the hypothesized bcam), then it might become attractive to model the appendage as an elastic continuum, writing partial differential equations of vibration, seeking normal modes of vibration, and truncating to a modest number of coordinates and a corresponding number of ordinary scalar differential equations. One must then return to those terms in Eqs. (31-35) which depend on deformation (noting Eqs. 7 and 8) and evaluate those terms as functions of the modal deformation coordinates, ignoring second degree terms in deformation variables.

In most cases, it will prove more feasible to adopt for an elastic appendage a finite-element model such as that described in Section $\mathbf{I}$. The equations of small vibratory deformation can then be recorded from Ref. $1^{4}$ (see Eq. 64), and the appropriate coordinate transformation can be adopted from Ref. 1 (see Eq. 74). The vector $\mathrm{c}^{k}$ in Eq. (8) is then replaced by $\mathrm{c}$ as recorded in Ref. 1, Eq. (43), with $\mathbf{e} \equiv 0$ and summation extending only over the $k$ th substructure. Although the dyadic $\mathbf{J}^{k}$ in Eq. (7) and the integral

$$
\frac{i d}{d t} \int_{a_{k}} \mathbf{p} \times \stackrel{\circ}{\mathbf{p}} d m
$$

are not provided in Ref. 1, which treats the distributed-mass finite-element model with nodal bodies, they are available in Ref. 5 for the special case with all mass concentrated in the nodal bodies; see Ref. 5, Eq. (126) for $\mathbf{J}^{k}$ and Eq. (127) for $\mathbf{J}^{k}$, and see Eq. (112) or Eq. (114) for

$$
\frac{{ }^{i} d}{d t} \int_{\alpha_{\mathrm{k}}} \mathbf{p} \times \stackrel{\circ}{\mathbf{p}} d m
$$
"Note that the vector called $\mathbf{X}$ in Ref, 1 must be interpreted for the rth appendage of the present
paper as $\mathbf{X}+\boldsymbol{\rho}^{r}$, with $\rho^{r}$ cxpanded as in Eq. (19). 
recognizing in each case that the superscript $k$ indicates restriction to the $k$ th substructure, and setting $\Omega^{a}$ and $\xi$ as found in Ref. 5 identically to zero. In each case the indicated expressions involve deformation variables which must be subjected to coordinate transformations and truncations, as indicated in Refs. 1 and 5.

If it pleases the analyst, he can conceal the complexities of modeling the elastic appendage by simply adopting a symbolic model in terms of mode shapes and natural frequencies. Then he can rather easily record the equations of vibration (probably using Lagrange's equations with modal coordinates for generalized coordinates), and he can evaluate the deformation-dependent terms in Eqs. (31-33) in terms of unspecified mode shapes and frequencies. Of course he must face the problems of adopting an appendage model and determining its modal characteristics when he wants to face any real problem.

\section{Matrix Equations}

This section illustrates the transition from the preceding vector-dyadic equations to an alternative matrix form which is more explicit and therefore more suitable for programming for digital computer numerical integration. To obtain explicit results, we must make a commitment to a particular appendage model, sacrificing some of the generality of the vector-dyadic equations. In what follows, we confine attention to the special case of the finite element appendage model in Ref. 1 for which all mass is concentrated in the $n$ nodal bodies of the system (with no distributed mass for the internodal elastic elements). All deformations from a nominal appendage state are assumed arbitrarily small, so that terms above the first degree in these deformations and the corresponding deformation rates can be neglected. Moreover, we assume that only the reference body $b_{0}$ contains rotors, and these number three, establishing an orthogonal triad whose axes parallel $\mathbf{b}_{1}^{0}, \mathbf{b}_{2}^{0}$, and $\mathbf{b}_{3}^{0}$. The restrictions adopted in this section reduce the number of terms appearing in the final equations of motion somewhat, but (as shown in Ref. 1) they do not change the structure of the equations, so that the special case treated here remains representative of the general case of small-vibration appendages. (Large-amplitude appendage vibrations, which are accommodated in the preceding vector-dyadic equations, would produce matrix equations quite different from those to be recorded here.)

The vector-dyadic equations to be written in matrix form are Eqs. (9) and (31-35), in combination with Eq. (64) of Ref. 1, with various results substituted from Refs. 1 and 5 .

Equation (9) may be rewritten in terms of Defs. 8, 15, and 28 as

$$
\{\mathbf{i}\}^{T} C^{T} F=\{\mathbf{i}\}^{T} m \ddot{X}
$$

which implies the matrix equation

$$
F=m C \ddot{X}
$$

The time history of the direction cosine matrix $C$ is cstablished by the Euler-Poisson kinematic equation (see Ref. 5, Eq. (44), for tilde convention).

$$
\dot{C}=-\varpi^{0} C
$$


Skipping next to the rotor equations and noting the restriction to the three orthogonal rotors in $b_{0}$ (so that $k=0$ only, and $\mathbf{b}^{0 s}=\mathbf{b}_{s}^{0}$ for $s=1,2,3$ ), we have from Eq. (36) the three scalar equations

$$
\tau_{08}^{R}=\mathscr{D}_{O s}\left(\ddot{\psi}_{O s}+\dot{\omega}_{s}^{0}\right)
$$

or the implied matrix equation

$$
\tau^{k}=\mathscr{D}\left(\ddot{\psi}+\dot{\omega}^{0}\right)
$$

where $\psi \triangleq\left[\psi_{01} \psi_{02} \psi_{08}\right]$ and $\mathscr{Z}$ is a diagonal matrix with diagonal elements $\mathscr{L}_{01}, \mathscr{L}_{02}$, and $\mathscr{Z}_{03}$.

The vector rotational equations are easily written in matrix form with the definition

$$
\mathbf{W}^{k} \triangleq \mathbf{W}^{k} \cdot\left\{\mathbf{b}^{k}\right\}
$$

since then, in terms of the direction cosine matrices in Def. 31, we can write Eqs. (31) and (32) as

or simply

$$
\sum_{\mathbf{k} \in \mathbb{B}}\left\{\mathbf{b}^{k}\right\}^{T} W^{k}=\left\{\mathbf{b}^{0}\right\}^{T} \sum_{k \in \mathbb{B}} C^{0 k} W^{k}=0
$$

and

$$
\sum_{k \in \mathbb{B}} C^{0 k} W^{k}=0
$$

or

$$
\tau_{s}+g^{s^{T}}\left\{\mathbf{b}^{s}\right\} \cdot \sum_{k \in \mathcal{G}} \varepsilon_{8 k}\left\{\mathbf{b}^{k}\right\}^{T} W^{k}=0
$$

$$
\tau_{s}+g^{s^{2}} \sum_{k \in \mathcal{S}} \varepsilon_{s k} C^{s k} W^{k}=0
$$

Equations (40) and (4I) require $W^{k}$, which is available from Eq. (33) when written in the form

$$
\begin{aligned}
& \left\{\mathbf{b}^{k}\right\}^{T} W^{k}=\left\{\mathbf{b}^{k}\right\}^{T} \mathbf{T}^{k}+\sum_{\boldsymbol{r} \in \mathbb{B}}\left\{\mathbf{b}^{k}\right\}^{T} D^{k r} \times\left\{\mathbf{b}^{r}\right\}^{T} F^{r} \\
& +\left\{\mathbf{b}^{k}\right\}^{r^{r}} c^{k} \times\left(\frac{m_{k}}{m}\left\{\mathbf{b}^{0}\right\}^{T} F-\left\{\mathbf{b}^{k}\right\}^{r} \boldsymbol{F}^{k}\right) \\
& +\sum_{r \in \mathscr{B}} m_{r}\left\{\mathbf{b}^{k}\right\}^{r} D^{k r} \times\left\{\mathbf{b}^{r}\right\}^{r}\left[\ddot{c}^{r}+2 \widetilde{\omega}^{r} c^{r}+\widetilde{\omega}^{r} c^{r}+\widetilde{\omega}^{r} \widetilde{\omega}^{r} c^{r}\right] \\
& +m_{k}\left\{\mathbf{b}^{k}\right\}^{T} c^{k} \times \sum_{r \in \mathbb{B}}\left\{\mathbf{b}^{r}\right\}^{T}\left[\tilde{\tilde{\omega}}^{r} D^{r k}+\widetilde{\omega}^{r} \widetilde{\omega} D^{r k}\right]-\left\{\mathbf{b}^{k}\right\}^{T} \Phi^{k k} \dot{\omega}^{k} \\
& +\sum_{r \in \mathcal{B}-k}\left\{\mathscr { m } \left[D^{r k^{T}}\left\{\mathbf{b}^{r}\right\} \cdot\left\{\mathbf{b}^{k}\right\}^{T} D^{k r}\left\{\mathbf{b}^{k}\right\}^{T} U\left\{\mathbf{b}^{k}\right\}\right.\right. \\
& \left.\left.-\left\{\mathbf{b}^{r}\right\}^{T} D^{r k} D^{k r^{T}}\left\{\mathbf{b}^{k}\right\}\right] \cdot\left\{\mathbf{b}^{r}\right\}^{T} \dot{\phi}^{r}\right\} \\
& +\infty \sum_{r \in \mathcal{B}-\mathbf{k}}\left[\left\{\mathbf{b}^{k}\right\}^{T} D^{k r} \times\left\{\mathbf{b}^{r}\right\}^{T} \widetilde{\omega}^{r} \widetilde{\omega}^{r} D^{r k}\right]-\left\{\mathbf{b}^{k}\right\}^{T} \tilde{\omega}^{k} \Phi^{k k} \omega^{k} \\
& -\left\{\mathbf{b}^{k}\right\}^{T} \dot{h}^{k}-\left\{\mathbf{b}^{k}\right\}^{T} \widetilde{\omega}^{k} h^{k}-\left\{\mathbf{b}^{k}\right\}^{T} \int_{\boldsymbol{a}_{k}} \tilde{p} \ddot{p} d m-\left\{\mathbf{b}^{k}\right\}^{T} \widetilde{\omega}^{k} \int_{a_{k}} \tilde{p} \dot{p} d m
\end{aligned}
$$


since then

$$
\begin{aligned}
& W^{k}=\left\{\mathbf{b}^{k}\right\} \cdot\left\{\mathbf{b}^{k}\right\}^{T} W^{k} \\
& =T^{k}+\sum_{r \in \mathcal{B}} \widetilde{D}^{k r} C^{k r} F^{r}+\widetilde{c}^{k}\left(\frac{m_{k}}{m} C^{k v} F-F^{k}\right) \\
& +\sum_{r \in \mathscr{B}} m_{r} \widetilde{D}^{k r} C^{k r}\left(\ddot{c}^{r}+2 \widetilde{\omega}^{r} \dot{c}^{r}+\widetilde{\tilde{\omega}}^{r} c^{r}+\widetilde{\omega}^{r} \widetilde{\omega}^{r} c^{r}\right) \\
& +m_{k} \widetilde{c}^{k} \sum_{r \in \Phi} C^{k r}\left(\tilde{\omega}^{r} D^{r k}+\tilde{\omega}^{r} \omega^{r} D^{r k}\right)-\Phi^{k k} \dot{\omega}^{k}-\dot{\Phi}^{k k} \omega^{k} \\
& +m \sum_{r \in \mathscr{B}-\mathbf{k}}\left[\left(D^{r k T} C^{r k} D^{k r} U-C^{k r} D^{r k} D^{k r T}\right) C^{k r} \dot{\omega}^{r}\right. \\
& +\tilde{D}^{k r} C^{\left.k+\tilde{\omega}^{\top} \widetilde{\omega}^{r} D^{r k}\right]} \\
& -\widetilde{\omega}^{k} \Phi^{k k} \omega^{k}-\dot{h}^{k}-\widetilde{\omega}^{k} h^{k}-\int_{a_{k}} \tilde{p} \ddot{p} d m-\widetilde{\omega}^{k} \int_{a_{k}} \tilde{p} \dot{p} d m
\end{aligned}
$$

Even with the substitution of Eq. (42) into Eqs. (40) and (41), the matrix equations are not in their final form, since the variables $\omega^{k}, \omega^{k}, h^{k}, c^{k}, \Phi^{k k}$ the direction cosine matrices such as $C^{k r}$, and the integrals over $\boldsymbol{a}_{k}$ are not yet sufficiently explicit. These deficiencies are rectified by substituting matrix versions of Eqs. (34), (35), (37), (8), and (7), together with new expressions for $C^{k r}$ and expressions for the integrals obtained from Ref. 5. Specifically, from Eqs. (34) and (35), we find

$$
\omega^{k} \triangleq\left\{\mathbf{b}^{k}\right\} \cdot \omega^{k}=\left\{\mathbf{b}^{k}\right\} \cdot\left[\left\{\mathbf{b}^{0}\right\}^{T} \omega^{0}+\sum_{r \in \mathcal{P}} \varepsilon_{r k} \dot{\gamma}_{r}\left\{\mathbf{b}^{r}\right\}^{T} g^{r}\right]
$$

or

$$
\omega^{k}=C^{k 0} \omega^{0}+\sum_{r \notin \mathcal{P}} \varepsilon_{r k} \dot{\gamma}_{r} C^{k r} g^{r}
$$

and

$$
\begin{aligned}
\dot{\omega}^{k} \triangleq & \left\{\mathbf{b}^{k}\right\} \cdot \dot{\omega}^{k}=\left\{\mathbf{b}^{k}\right\} \cdot\left\{\mathbf{b}^{0}\right\}^{r} \dot{\omega}^{0} \\
& +\sum_{r \in \mathcal{S}} \varepsilon_{r k}\left[\ddot{\gamma}_{r}\left\{\mathbf{b}^{k}\right\} \cdot\left\{\mathbf{b}^{r}\right\}^{T} g^{r}+\left\{\mathbf{b}^{k}\right\} \cdot\left\{\mathbf{b}^{0}\right\}^{T} \omega^{0} \times\left\{\mathbf{b}^{r}\right\}^{T} g^{r}\right. \\
& \left.+\left\{\mathbf{b}^{k}\right\} \cdot \sum_{s \in \mathcal{S}} \varepsilon_{s r} \dot{\gamma}_{8}\left\{\mathbf{b}^{s}\right\}^{T} g^{s} \times\left\{\mathbf{b}^{r}\right\}^{T} \mathbf{g}^{r}\right]
\end{aligned}
$$

or

$$
\dot{\omega}^{k}=C^{k 0} \dot{\omega}^{0}+\sum_{r \varepsilon \mathscr{Q}} \varepsilon_{r k}\left[\ddot{\gamma}, C^{k r} g^{r}+C^{k o} \widetilde{w}^{0} C^{0 r} g^{r}+\sum_{s \in \mathscr{Q}} \varepsilon_{s i} \dot{\gamma}_{s} C^{k s} \widetilde{g}^{s} C^{s r} g^{r}\right]
$$

For the special case at hand, Eq. (37) yields $h^{k} \equiv 0$ for $k \neq 0$ and

$$
h^{0}=\left\{\mathbf{b}^{0}\right\} \cdot \mathbf{h}^{0}=\not{\not} \dot{\psi}
$$

as these symbols are defined following Eq. (39). 
For the nodal body system to which this section is limited, Eq. (8) becomes a special case of Eq. (45) of Ref. 1 or a variant of Eq. (60) of Ref. 5. Specifically, we have

$$
c^{k}=-\sum_{\delta=1}^{n_{k}} \frac{m_{u}}{m_{k}} u^{s}
$$

where appendage $\boldsymbol{\alpha}_{k}$ has been idealized as $n_{k}$ nodal bodies interconnected by massless elastic structure, with $m_{s}$ the mass of nodal body $s$ and $u^{*} \stackrel{\Delta}{=}\left[\begin{array}{llll}u_{1}^{s} & u_{2}^{s} & u_{3}^{s}\end{array}\right]^{T}$ given by $u^{s} \triangleq\left\{\mathbf{b}^{k}\right\} \cdot \mathbf{u}^{s}$ with $\mathbf{u}^{s}$ the displacement of the body $s$ relative to $k_{k}$ from the position occupied in the nominal state.

Recalling from Defs. (38), (37), and (24) that

$$
\Phi^{k k}=\boldsymbol{K}^{k}+J^{k}
$$

and noting that $K^{k}$ is constant and $J^{k}$ is available from Fq. (7) as the variable matrix

$$
J^{k}=\int_{d_{k}}\left(p^{T} p U-p p^{p}\right) d m
$$

one can, for the presently adopted appendage model, evaluate $J^{k}$ as (sec Rcf. 5, Eq. (126))

$$
J^{k}=\bar{J}^{k}+\sum_{s=1}^{n_{i c}}\left\{m_{s}\left[2\left(R^{k}+r^{s}\right)^{T} u^{s} U-\left(R^{k}+r^{s}\right) u^{s^{T}}-u^{s}\left(R^{k}+r^{s}\right)^{T}\right]+\bar{\beta}^{s} I^{s}-I^{s} \bar{\beta}^{s}\right\}
$$

where $\bar{J}^{k}$ is the nominal (constant) value of $J^{k}$, and $I^{s}$ is the constant incrtia matrix of the sth nodal body in its own body-fixed vector basis $\left\{n^{s}\right\}^{k}$, where, in the nominal state, $\left\{\mathbf{n}^{s}\right\}^{k}=\left\{\mathbf{b}^{k}\right\}$. Herc we use $\boldsymbol{u}^{s}$ for $\left(\boldsymbol{u}^{s}\right)^{k}$, as in Def. 21 .

In combination, Eqs. (47) and (48) provide

$\dot{\Phi}^{k k}=\sum_{s=1}^{n_{k}}\left\{m_{s}\left[2\left(\mathbf{R}^{k}+\boldsymbol{r}^{s}\right)^{T} \dot{u}^{s} U-\left(\boldsymbol{R}^{k}+r^{s}\right) \dot{u}^{s^{T}}-\dot{u}^{s}\left(\boldsymbol{R}^{k}+r^{s}\right)^{r}\right]+{\tilde{\dot{\beta}^{s}}}^{s}-\mathbf{I}^{s} \tilde{\dot{\beta}}^{s}\right\}$

An expression for the direction cosine matrix $C^{k N_{k}}$ (where $k \in \mathbb{R}$ and $N_{k}$ is a network element from Def. 32) may be found from Ref. 7 to be

$$
C^{k N_{k}}=U \cos \gamma_{k}-\tilde{g}^{k} \sin \gamma_{k}+g^{k} g^{k T}\left(1-\cos \gamma_{k}\right)
$$

and, by transposition,

$$
C^{N} k^{k}=U \cos \gamma_{k}+\tilde{g}^{k} \sin \gamma_{k}+g^{k} g^{k T}\left(1-\cos \gamma_{k}\right)
$$

For arbitrary $r, j \in \mathscr{B}$, the direction cosine matrix $C^{r j}$ can be constructed as a product of direction cosine matrices relating contiguous bodies (as given by Eqs. 50 and 51). 
The required product can be written symbolically as

$$
C^{r j}=\Pi_{k=r}^{N_{j r}} C^{k N_{k j}}
$$

where the symbol $\prod_{k=r}^{N_{j r}}$ is understood to imply the following algorithm:

(1) Define $p \triangleq N_{r j}$ and construct $C^{r p}$ from Eq. (50) if $r>p$ and from Eq. (51) if $r<p$.

(2) Define $q \triangleq N_{p j}$ and construct $C^{p q}$ from Eq. (50) if $p>q$ and from Eq. (51) if $p<q$.

(3) Proceed until an integer $u$ emerges such that $j \triangleq N_{u_{j}}$, finally constructing $C^{u j}$ from Eq. (50) if $u>i$ and from Eq. (51) if $u<i$.

(4) Multiply the matrices obtained in the sequence

$$
C^{r j}=C^{r p} C^{p q} \cdots C^{u j}
$$

Finally, Eq. (42) requires more explicit expressions for the integrals over the appendage $a_{k}$. For the special case of elastic appendages idealized as interconnected nodal bodies, the appropriate expressions can be found in vector form in Eq. (114) of Ref. 5. The corresponding matrix equations are

$$
\begin{aligned}
& -\int_{m_{k}} \tilde{p} \ddot{p} d m-\widetilde{\omega}^{k} \int \tilde{p} \dot{p} d m=-\left(\widetilde{\omega}^{k} R^{k}\right)^{\sim} \sum m_{s} \dot{u}^{s} \\
& -\tilde{R}^{k}\left(\sum m_{s} \ddot{u}^{s}+\widetilde{\omega}^{k} \sum m_{s} \dot{u}^{s}\right)-\Sigma\left(\widetilde{\omega}^{k} r^{8}\right)^{\sim} m_{s} \dot{u}^{s} \\
& -\sum \widetilde{r}^{s}\left(m_{s} \ddot{u}^{s}+\widetilde{\omega}^{k} m_{s} \dot{u}^{s}\right)-\sum I^{s}\left(\ddot{\beta}^{s}+\widetilde{\omega}^{k} \dot{\beta}^{s}\right) \\
& -\widetilde{\omega}^{k} \sum I^{s} \dot{\beta}^{s}+\sum I s \widetilde{\omega}^{k} \dot{\beta}^{s} \\
& =-\Sigma\left(\widetilde{R}^{k}+\tilde{r}^{s}\right) m_{s} \ddot{u}^{s}-\widetilde{\omega}^{k} \sum\left(\widetilde{R}^{k}+\tilde{r}^{s}\right) m_{s} \dot{u}^{s} \\
& -\Sigma\left[I^{s}\left(\ddot{\beta^{s}}+\widetilde{\omega}^{k} \dot{\beta}^{s}\right)-\widetilde{\boldsymbol{\omega}}^{k} I^{s} \dot{\beta}^{s}+I^{s} \widetilde{\omega}^{k} \dot{\beta}^{8}\right]
\end{aligned}
$$

where $\Sigma$ implies summation over the range from $s=1$ to $s=n_{k}$, and the superscript $k$ has been dropped from nodal body variables in the $k$ th appendage (such as $u^{8}$, which replaces $\left.\left(u^{s}\right)^{k}\right)$. In developing Eq. (5.3), the identity $(\widetilde{a} b)^{\sim}=\tilde{a} \tilde{b}-\tilde{b} \tilde{a}$ has been utilized.

In summary, in order to obtain the explicit matrix equations defining substructure rotations, one must combine Eqs. (42-53) into Eqs. (40) and (4I). The result is a set of $n+3$ scalar equations, which can be collected together with the three rotor equations (Eq. 39) and the three vehicle translation equations (Eq. 38a) and their kinematic equations (Eq. 38b) to comprise a set which is complete except for control system equations and the equations of vibration of each of the $n+1$ append- 
ages. The latter set can be obtained for a distributed-mass finite-element model from Eq. (64) of Ref. 1, and for the special case of the concentrated-mass (nodal body) finite-element model either from Eq. (64) of Ref. 1 or from Eq. (95) of Ref. 5 (correcting the last algebraic sign within the braces on the right side of Eq. (95) by changing - to + ), recognizing that what is recorded as $\Theta \ddot{X}$ in those equations must be replaced by the inertial acceleration of the mass center of the corresponding substructure in the local vector basis, which for substructure $a_{k}$ is given by

$$
\begin{aligned}
C \ddot{X}+\left\{\mathbf{b}^{k}\right\} \cdot \ddot{\mathbf{\rho}}^{k}= & C \ddot{X}+\left\{\mathbf{b}^{k}\right\} \cdot\left[\sum_{r \in \mathbb{B}}\left(\ddot{\mathbf{D}}^{r k}+\frac{m_{r}}{m} \ddot{\mathbf{c}}^{r}\right)-\ddot{\mathbf{c}}^{k}\right] \\
= & C \ddot{X}-\left(\ddot{c}^{k}+\tilde{\tilde{\omega}}^{k} c^{k}+2 \widetilde{\omega}^{k} \dot{c}^{k}+\widetilde{\omega}^{b} \tilde{\omega}^{k} c^{k}\right) \\
& +\sum_{r e \mathscr{B}} C^{k r}\left[\left(\widetilde{\tilde{\omega}}^{r}+\widetilde{\omega}^{r} \widetilde{\omega}^{r}\right)\left(D^{r k}+\frac{m_{r}}{m} c^{r}\right)+\frac{m_{r}}{m}\left(\ddot{c}^{r}+2 \widetilde{\omega}^{r} \dot{c}^{r}\right)\right]
\end{aligned}
$$

where Eq. (19) has been utilized for the expansion of $\ddot{\rho}^{k}$.

Typically the number of nodes $n_{k}$ in a single finite-element model of an elastic appendage is so great that the proposed combination of nodal body vibration equations with substructure rotation equations, vehicle translation equations, rotor equations, and control system equations would be prohibitive in size for numerical integrations. Thus it is to be understood at the outset that the nodal body vibration equations will provide the basis for a transformation to distributed or modal coordinates for appendage deformations, and that most of the modal coordinates will be deletcd from consideration by truncating the matrix of deformation variables. By means of this transformation, all variables labeled $u^{s}$ and $\beta^{s}$ in Eqs. (46-53) are replaced by appropriate combinations of new modal deformation variables. Thus the equations actually to be programmed for digital computer numerical integration are not precisely those obtained from Eqs. (38-53) of this report plus Eq. (64) of Ref. 1 (or Eq. 95 of Ref. 5), but instead the transformed and truncated versions of these equations. Because questions of transformation, truncation, and synthesis of the resulting composite matrix equations have been treated extensively in Refs. 1, 5 , and 8 , these matters will not be considered further here.

\section{Summary}

By combining Eqs. (9), (31), (32), and (36) with Eq. (64) of Ref. 1, one can obtain a generic statement of a minimum dimension set of equations of motion of a system of $n+1$ rigid bodies interconnected by $n$ line hinges, with a set of axisymmetric rotors and a distributed-mass finite-element model of an elastic appendage attached to each rigid body. In order for these equations to stand alone as a complete formulation of the problem, one must substitutc the auxiliary equations $(33-35)$ and (37), as well as certain specified equations from Ref. 5, into Eqs. (31) and (32), and Eq. (64) of Ref. 1 must be composed from the underlying equations of that reference (Eqs. 46, 53, and 62). Moreover, the total system of equations must for practical utilization be written in matrix form and the appendage deformations subjected to modal coordinate transformations and truncations, as these procedures are described in Refs. 1 and 5 and illustrated in part for a special case in the preceding section of this report. 
Although one must not underestimate the labors of proceeding from the vectordyadic equations in this report to a generic computer program for integrating these equations, this does appear to be a feasible task. Once the program is completed, it will have sufficient generality to encompass several spacecraft simulations for which specific numerical integration computer programs have been written in the past (Refs. 8-10). Experience with these programs provides reasonable assurance that the generic program proposed here will find application as a practical tool for the simulation of complex modern spacecraft.

\section{References}

1. Likins, P. W., "Finite Element Appendage Equations for Hybrid Coordinate Dynamic Analysis," Int. J. Solids Struct,, Vol. 8, pp. 709-731, 1972. (Also available in expanded form as Technical Report 32-1525, Jet Propulsion Laboratory, Pasadena, Calif., Oct. 15, 1971.)

2. Hooker, W. W., and Margulies, G., "The Dynamical Attitude Equations for an n-Body Satellite," J. Astronaut. Sci., Vol. 12, pp. 123-128, 1965.

3. Hooker, W. W., "A Set of $r$ Dynamical Attitude Equations for an Arbitrary $N$-Body Satellite Having $r$ Rotational Degrees of Freedom," AIAA J., Vol. 8, pp. 1205-1207, 1970.

4. Roberson, R. E., and Wittenburg, J., “A Dynamical Formalism for an Arbitrary Number of Interconnected Rigid Bodies, With Reference to the Problem of Satellite Attitude Control," Proc. 3rd Int. Congress of Automatic Control (London, 1966), Butterworth and Co., Ltd., London, 1967.

5. Likins, P. W., Dynamics and Control of Flexible Space Vehicles, Technical Report 32-1329, Rev. I, Jet Propulsion Laboratory, Pasadena, Calif., Jan. 15, 1970.

6. Likins, P.W., Barbera, F.J., and Baddeley, V., "Mathematical Modeling of Spinning Elastic Bodies for Modal Analysis," submitted to AIAA J.

7. Likins, P. W., and Fleischer, G. E., Large Deformation Modal Coordinates for Nonrigid Vehicle Dynamics, Technical Report 32-1565, Jet Propulsion Laboratory, Pasadena, Calif., Nov. 1, 1972.

8. Gale, A. H., and Likins, P. W., "Influence of Flexible Appendages on Dual-Spin Spacecraft Dynamics and Control," J. Spacecraft Rockets, Vol. 7, pp. 1049-1056, 1970 .

9. Likins, P. W., and Fleischer, G. E., "Results of Flexible Spacecraft Attitude Control Studies Utilizing Hybrid Coordinates," J. Spacecraft Rockets, Vol. 8, pp. 264-273, 1971.

10. Fleischer, G. E., and McGlinchey, L. F., "Viking Thrust Vector Control Dynamics Using Hybrid Coordinates to Model Vehicle Flexibility and Propellant Slosh," AAS/AIAA Paper 71-348, presented at the Astrodynamics Specialists' Conference, Ft. Lauderdale, Fla., American Astronautical Society, 1971. 